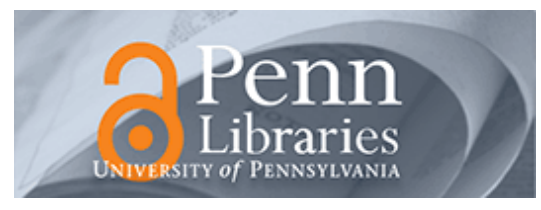

University of Pennsylvania

ScholarlyCommons

$6-5-2012$

\title{
Procurement in Supply Chains When the End-Product Exhibits the 'Weakest Link' Property
}

Stanley Baiman

University of Pennsylvania

Serguei Netessine

University of Pennsylvania

Howard Kunreuther

University of Pennsylvania

Follow this and additional works at: https://repository.upenn.edu/bepp_papers

Part of the Business Commons, Economics Commons, and the Public Affairs, Public Policy and Public Administration Commons

\section{Recommended Citation}

Baiman, S., Netessine, S., \& Kunreuther, H. (2012). Procurement in Supply Chains When the End-Product Exhibits the 'Weakest Link' Property. SSRN's eLibrary, http://dx.doi.org/10.2139/ssrn.2077640

This paper is posted at ScholarlyCommons. https://repository.upenn.edu/bepp_papers/8

For more information, please contact repository@pobox.upenn.edu. 


\title{
Procurement in Supply Chains When the End-Product Exhibits the 'Weakest Link' Property
}

\author{
Abstract \\ We consider a supply chain with one manufacturer who assembles an end-product using multiple \\ outsourced parts. The end-product exhibits the "weakest-link" property, such that if any of its component \\ parts fails, the end-product fails. The supplier of each component part can improve the (uncertain) quality \\ of her parts by exerting costly effort that is unobservable to the manufacturer and is non-contractible. We \\ analyze three possible contractual agreements between the manufacturer and suppliers: Acceptable \\ Quality Level (AQL), Quality-Based Incentive Pricing (Q-Pricing) and Group Warranty. Under AQL, the \\ manufacturer inspects all incoming parts, but establishes different quality thresholds and pays the \\ suppliers different amounts for achieving the different thresholds. Under Q-Pricing, the manufacturer also \\ inspects all incoming parts but pays each supplier a constant amount for each good part. Under Group \\ Warranty there is no testing of the individual parts; instead all suppliers are responsible for any failed end- \\ product. We compare the efficiency of these three contractual arrangements as a function of the \\ exogenous variables.

\section{Disciplines} \\ Business | Economics | Public Affairs, Public Policy and Public Administration
}




\title{
Procurement in Supply Chains when the End-Product Exhibits the "Weakest Link" Property" Unabridged
}

\author{
Stanley Baiman \\ Serguei Netessine ${ }^{2}$ \\ Howard Kunreuther \\ The Wharton School \\ University of Pennsylvania \\ Philadelphia, PA 19104
}

February, 2004

\begin{abstract}
We consider a supply chain with one manufacturer who assembles an end-product using multiple outsourced parts. The end-product exhibits the "weakest-link" property, such that if any of its component parts fails, the end-product fails. The supplier of each component part can improve the (uncertain) quality of her parts by exerting costly effort that is unobservable to the manufacturer and is non-contractible. We analyze three possible contractual agreements between the manufacturer and suppliers: Acceptable Quality Level (AQL), Quality - Based Incentive Pricing (Q - Pricing) and Group Warranty. Under AQL, the manufacturer inspects all incoming parts, but establishes different quality thresholds and pays the suppliers different amounts for achieving the different thresholds. Under Q Pricing, the manufacturer also inspects all incoming parts but pays each supplier a constant amount for each good part. Under Group_Warranty there is no testing of the individual parts; instead all suppliers are responsible for any failed end-product. We compare the efficiency of these three contractual arrangements as a function of the exogenous variables.
\end{abstract}

\footnotetext{
${ }^{1}$ We are grateful to Gerard Cachon, Madhav Rajan and seminar participants at the Wharton School for comments on the earlier version of this paper.

${ }^{2}$ Corresponding author, netessine@wharton.upenn.edu, (215) 573 3571, this paper is available at http://www.netessine.com.
} 


\section{Introduction}

End-product manufacturers often outsource the production of many of the parts or even whole sub-assemblies that go into the manufacture of their end-products. ${ }^{3}$ As a result, the quality of their end-products is dependent on the quality of the parts supplied by others. The need to ensure the quality of these sub-contracted parts is especially important when one defective part provided by a supplier has the potential to cause the failure of the entire end-product. ${ }^{4}$ In a world of no supplier incentive problems, i.e., one in which suppliers will costlessly implement any decision rule specified by the manufacturer, as the number of parts increases, holding all else fixed, the probability that the end-product fails increases. But, what additional effects does the number of parts have in the presence of incentive problems involving the suppliers? Moreover, what effect does the number of parts that go into the end-product have on the performance of different types of contractual relationships between the manufacturer and suppliers? ${ }^{5}$ These questions are the focus of our paper.

We consider a stylized game-theoretic model in which a single manufacturer (hereafter referred to as $h e$ ) produces a good which is made up of $n$ different parts or components, $i=1,2, \ldots, n$. Part $i$ is produced and supplied only by supplier $i$ (each hereafter referred to as she). Each supplier agrees to supply a batch of units of her part to the manufacturer. Each end-product is composed of one part from each supplier. Some of the parts supplied may be defective. Each supplier determines her own yield rate of non-defective parts based on her own cost-of-yield which can take one of two possible realizations: high- and low-cost.

At the time of contracting, no one knows the cost-of-yield realizations, but everyone agrees on the feasible set of realizations and their probability distributions. Thus, contracting takes place under symmetric but incomplete information. After the contracts have been agreed upon, each supplier privately observes her own cost-of-yield realization. The yield rate chosen by each supplier is not observed by any other party. Thus, each supplier chooses her yield rate based on her private information so as to maximize her own expected profit rather than the manufacturer's. This is the

\footnotetext{
${ }^{3}$ Pratt \& Whitney, GE and Rolls Royce manufacture most airplane engines for Boeing and Airbus. Maxtor, Western Digital and Seagate manufacture most computer hard drives for Dell, Compaq and IBM.

${ }^{4}$ NASA estimated that in the Challenger, there were approximately 4,686 critical parts, such that if any one of them failed, the entire mission would fail (Pate-Cornell and Dillon, 2001). A comparable, but less extreme, example is a product like a VCR where if one part fails it is cheaper to replace the entire unit than to replace the defective part.

${ }^{5} \mathrm{We}$ do not, however, address the more basic question of when firms would choose to insource vs. outsource the production of parts. See Monteverde and Teece (1982) for an analysis of this issue in the US automobile industry.
} 
suppliers' incentive problem faced by the manufacture. A key feature of the model is the "weakestlink" property of the end-product: failure of any single component part causes the failure of the endproduct that is assembled by the manufacturer. The manufacturer wishes to design a contractual agreement with the suppliers that will maximize his profit. We analyze and compare three such contracts described below.

Many companies inspect incoming parts to ensure their conformity (see, e.g., Bossert 1994, page 65). We first consider a situation in which the manufacturer, costlessly and perfectly inspects all incoming parts, specifies threshold(s) of acceptable yield rates of parts and pays different amounts to suppliers who achieve different acceptable yield rates. This is often referred to as "Acceptable Quality Level" contracting (hereafter referred to as AQL, see Windham (1995)). We characterize the optimal AQL contract and analyze the distortion in the suppliers' yields arising from the underlying incentive problem.

We also show that, depending on problem parameters, two different contract forms can emerge. In the first, referred to as a Separating solution, each supplier's contract specifies multiple acceptable yield rates, one for each possible supplier cost realization. In the second, referred to as a Pooling solution, each supplier's contract specifies only one acceptable yield rate, regardless of the supplier's cost realization.

We next consider a different contract in which the manufacturer conducts a costless and perfect inspection of all parts and pays each supplier a constant amount for each good part and nothing for a defective one. This type of contract is often called "Quality - Based Incentive Pricing” (hereafter referred to as Q - Pricing), see Windham (1995). Notice that with this contract, the manufacturer pays for the good parts but does not specify a desired yield rate. We again analyze the distortions in the suppliers' resulting yield rates arising from the underlying incentive problems.

The Q - Pricing and AQL contracts depend on the manufacturer's ability to test the incoming parts. Another possibility is not to test incoming parts, but to contract on the failure of the endproduct. These are typically referred to as warranty contracts. If the failure of the end-product can be traced to the failure of a specific part, the same Q - Pricing and AQL contracts can still be written. ${ }^{6}$ However, these contracts are not feasible if it is too costly (or impossible) to assign blame to a

\footnotetext{
${ }^{6} \mathrm{GM}$ writes these types of parts-supply contracts. To facilitate this process, GM gives its 30,000 suppliers access to dealer warranty and repair database (Smith 1997). Lear, a major car seat manufacturer, writes these types of parts-supply contracts with its mold suppliers (Pryweller 1999).
} 
particular part based on an end-product failure. ${ }^{7}$ For this reason we examine a "Group Warranty" contract in which all suppliers bear the cost of an end-product failure, regardless of whose part caused the failure. ${ }^{8}$ Again, the resulting distortions in the suppliers' yield rates arising from underlying incentive problems are analyzed.

The remainder of the paper is organized as follows. In Section 2 we discuss the related literature. In Section 3 we lay out the model, discuss the assumptions and derive the solution when there are no supplier incentive problems. The AQL and Q - Pricing contracts are analyzed in section 4 and the Group Warranty contract in Section 5. We compare these three contracts in terms of expected profits to the manufacturer, suppliers and the supply chain in Section 6. Section 7 concludes the paper.

\section{Literature Survey}

The product design and development literature discusses product architecture including the number, design and interaction of product components primarily from an engineering/design perspective (Ulrich 1995, Ulrich and Eppinger 2000). However, such design decisions may also affect the incentives of the of the component parts suppliers. The incentive implications of the number of product components are the focus of this paper.

Likewise, much of the traditional quality literature studies the design of optimal testing policies without considering incentive implications. Our paper is closely related to the recent contracting on quality literature in supply chain management, e.g., Reyniers and Tapiero (1995), Baiman et al. (2000, 2001), Lim (2001), Iyer et al. (2002), and Balachandran and Radhakrishnan (2003). These papers analyze a situation in which a manufacturer contracts with a single supplier for a single part, while we consider the case of contracting for multiple parts provided by multiple suppliers. We demonstrate how the number of parts affects the extent of the supplier incentive problems when the product exhibits the weakest link property. Other papers study contracting with multiple suppliers in supply chains but, unlike the present paper, do not focus on quality issues (e.g., see Cachon 2002).

\footnotetext{
${ }^{7}$ A recent example of such a case is the Ford/Firestone battle over the cause of the rollover accidents involving the Ford Explorer (see Pinedo et al. 2003).

${ }^{8}$ Contracts that allow the manufacturer to reward the suppliers based on both the outcome from testing parts and external failure are outside the scope of this paper and hence are not analyzed.
} 
Our work is also related to the economics and accounting literature dealing with the agency problem (e.g., Alchian and Demsetz 1972, Holmstrom 1979 and Baiman and Rajan 2002). Our work differs from this literature in that we focus on situations with multiple agents and on a more specific context (the weakest link relationship). A subset of the contracting literature does allow for multiple agents, but addresses issues that are different from ours. Itoh $(1991,1994)$ allows for multiple agents but addresses the issue of when there are benefits to allowing the agents to help each other. The mechanism design literature (Demski and Sappington 1984 and Ma 1988) examines the effect of contracts on the implicit coordination among agents. We do not address either of these issues. The paper in this literature closest to ours is Harris et al. (1982) which analyzes resource allocation within a multi-divisional firm. Like our paper, Harris et al. (1982) assumes that the divisions (suppliers in our paper) possess private information about their productivity, their actions are unobservable, and that the firm's output is the minimum of the outputs of its divisions (our weakest link assumption). However, Harris et al. (1982) assumes a linear production technology, that devisions communicate their cost observations to the manufacturer who then sets production schedules (in our model such communications are not possible), focuses on one specific contract, and does not address the effect of the number of divisions (parts in our context) on the efficiency of the firm.

In the experimental game theory literature a similar concern arises in coordination games where actions of the "weakest-link" player determine payoffs to all other players (see Cachon and Camerer 1996). Finally, our work is somewhat related to recent papers on interdependent security in which the incentive to invest in protective measures is reduced if other parties have not taken similar steps (see Kunreuther and Heal 2003, Heal and Kunreuther 2003).

\section{Assumptions, notation and solution without contracting problems}

\section{a. Assumptions and notation}

Our model consists of one risk-neutral manufacturer who assembles an end-product consisting of $n$ parts, $i=1, \ldots, n$. Each part $i$ is supplied only by the risk neutral supplier $i{ }^{9}$ Each of the $n$ suppliers provides the same fixed number of parts, $Z$, to the manufacturer. This fixed lot size delivered

\footnotetext{
${ }^{9}$ We restrict our attention to the case in which there is a separate supplier for each part. If different parts were supplied by the same supplier then she would internalize part of the externality, partially mitigating, but not eliminating, the incentive problem which we study.
} 
by the suppliers may result from a fixed-batch-size production technology and the ability to produce only one batch per period. ${ }^{10}$ An example of a fixed-batch-size production technology is that employed in computer chip-making where chips are made on a standard sized wafer. Each end-product assembled by the manufacturer requires one part from each supplier and each supplier provides a different part. ${ }^{11}$ The manufacturer's only job is to assemble the end-product from the parts delivered by the suppliers, and the manufacturer does so without affecting the probability of the end-product failing. A finished end-product will fail if any one of its parts is defective. Supplier $i$ can influence the probability of her parts failing by choosing the percentage of parts which she delivers which are not defective (i.e., her yield rate) and thus will not fail if assembled into an end-product. Supplier $i$ 's chosen yield rate when her cost-of-yield is $c_{i j}$ is represented by $0 \leq x_{i j} \leq 1$, where the index $j$ represents the cost-of-yield realization. The cost to supplier $i$ of delivering $Z$ units with a yield rate of $x_{i j}$ is $Z c_{i j} x_{i j}^{2}{ }^{12}$ We further assume that all suppliers are symmetric and that there are only two cost-ofyield realizations, $\mathrm{c}_{1}$ and $\mathrm{c}_{2}$, where $\mathrm{c}_{1}<\mathrm{c}_{2}<\infty$, occur with probabilities $\mathrm{p}$ and 1-p, respectively, and $0<\mathrm{p}<1$. Each supplier's cost realization is independent of the other suppliers' cost realizations. We will denote the manufacturer's expected profit as $\Pi$ and each supplier's expected profit as $\pi$.

The order of events and additional assumptions (further discussed in Section 7) are:

Step 1. The manufacturer announces contract terms to the suppliers (which are not re-negotiable expost). At this point, the suppliers have not yet observed their own cost realizations. The manufacturer and the suppliers share common beliefs as to the feasible set of cost realizations $\left(c_{1}\right.$ and $c_{2}$ ) and the probability distribution over the cost realizations ( $p$ and 1-p). If the manufacturer tests the incoming parts, he commits to the parts testing policy. Further, we assume that each supplier can only observe the outcome of her own test, and hence can only be compensated upon the outcome of that test.

Step 2. Each supplier privately observes her own cost-of-yield realization. A supplier cannot communicate her cost realization to the manufacturer or to other suppliers. This implies that the contract negotiated in Step 1 cannot specify that supplier $i$ 's compensation or desired yield

\footnotetext{
${ }^{10}$ This technology is referred to as lot-for-lot production, see, e.g., Hopp and Spearman, 2000. Without this assumption each supplier would then have to decide on both her yield rate and the number of parts to deliver to the manufacturer. ${ }^{11}$ In a separate study we investigate the situation in which the same part may be sourced from multiple suppliers (see Baiman and Netessine 2004).

${ }^{12}$ Notice that the chosen yield rate is non-stochastic but whether any particular part produced is defective or non-defective is uncertain. Supplier $i$ 's chosen yield rate and cost-of-yield incorporate any parts testing which she may do prior to shipping the parts to the manufacturer.
} 
rate be a function of any communications regarding supplier $j$ 's cost realization. At this point in the process, each supplier has the option of costlessly abrogating the contract negotiated in Step 1 and not delivering the promised parts. A supplier will only implement the contract negotiated in Step 1 if her expected profit from doing so (conditional on her observed cost realization) exceeds her outside opportunity cost (e.g., supplying parts to another manufacturer). That outside opportunity cost is assumed to be zero. This assumption is referred to as the supplier's Limited Liability Constraint. We assume that the manufacturer designs the contract so that each supplier, regardless of her cost realization, has the incentive to implement the contract negotiated in Step 1.

Step 3. Each supplier $i$, after having observed her cost realization $c_{i j}$, simultaneously chooses her $x_{i j}$. These choices are not observable by the other suppliers or by the manufacturer.

Step 4. The manufacturer receives the parts from all suppliers. If the manufacturer tests the parts before assembling the non-defective parts into the end-product he does so here; otherwise he assembles the end-products, tests them, and sells the non-defective ones, collecting revenue $R$ for each. Suppliers are compensated according to the terms of the contract.

In Step 1, the manufacturer commits to one of two different testing strategies (test all incoming parts or just test the end-product) and to one of three different contractual arrangements (AQL, Q Pricing or Group Warranty). In Section 4 we assume that the manufacturer commits to costlessly and perfectly testing each part received from each supplier and assemble finished products from the nondefective parts. To illustrate further, if there were two suppliers, each delivering $Z$ parts, and one chose a yield rate of $40 \%$ while the other chose a yield rate of $60 \%$, the manufacturer would only have enough non-defective parts to assemble $\mathrm{Z}$ x minimum $(.4,6)$ end-products. More generally, the manufacturer's revenue function with parts testing is $R Z \min \left(x_{1 \bullet}, x_{2}, \ldots ., x_{n}\right)$, where $x_{i \bullet}$ is supplier $i$ 's realized yield rate. Notice that we are assuming that, because of time, spoilage, fashion concerns, or other constraints, the manufacturer can neither ask a supplier to replace his parts which were found to be defective, nor inventory any unused non-defective parts for use at some later date. ${ }^{13}$

In Section 5 we assume that the manufacturer foregoes testing the incoming parts and assembles end-products from all of the parts delivered. Therefore, if as before there were two

\footnotetext{
${ }^{13}$ The qualitative results would continue to hold as long as there is a cost to the manufacturer of receiving defective parts, for example, there is a positive cost to replace a defective part or there is a cost to inventory excess parts.
} 
suppliers, each delivering $Z$ parts, and one chose a yield rate of $40 \%$ while the other chose a yield rate of $60 \%$, the manufacturer would assemble $Z$ end-products, but the expected number of non-defective end-products would be $\mathrm{Z}(.4)(.6)$. More generally, the manufacturer's revenue function is $R Z\left(x_{1 \bullet}\right)\left(x_{2} \bullet\right)().().\left(x_{n \bullet}\right)$. We are thus assuming that the end-product is tested after assembly and before it is sold, and only the non-defective end-products are sold for the price $R$ per unit. ${ }^{14}$ Notice that the different testing assumptions lead to very different objective functions for the manufacturer. Given the assumed structure of the problem, we can, without loss of generality, assume that $Z=1$ and refer to $x_{i j}$ as both the yield rate and the number of non-defective parts.

\section{b) Team solution (no incentive problems)}

We begin our analysis by studying the solution to the situation in which there are no incentive problems but there is still information asymmetry in the sense that suppliers' cost realizations are private. We call this the Team solution (similar to Marschak and Radner 1972) and use it as a benchmark to interpret the results from the three different contractual arrangements. Therefore, in succeeding sections the optimal contracts given the presence of incentive problems can be compared to the Team solution to determine the effect that incentive problems alone have on the optimal contract.

In the Team solution the manufacturer provides each supplier with directions of the form: "if $c_{1}$ is observed, choose yield $x_{1}^{T}$, if $c_{2}$ is observed, choose yield $x_{2}^{T}$ ". Each supplier will do as directed as long as she is no worse off by implementing the contract than by supplying some other manufacturer and earning her outside opportunity cost of zero. Thus, the payment to supplier with cost realization $c_{j}$ must be $T_{j}=c_{j}\left(x_{j}^{T}\right)^{2}$. Notice that the Team solution will be worse than First-Best because there is asymmetric information. Under First-Best, the manufacturer would base each supplier's yield on all $n$ cost realizations. However, in our problem each supplier knows only its own cost realization. This asymmetry of information causes a loss of efficiency in the Team solution, relative to First-Best, because of the resulting lack of coordination among the suppliers. However, because each supplier

\footnotetext{
${ }^{14}$ Alternatively, we could assume that the manufacturer charges $R$ per unit sold and returns the $R$ to the customer if the endproduct proves to be defective. The qualitative results would continue to hold if there was a reputational cost to the manufacturer of selling defective end-products.
} 
will implement whatever yield strategy the manufacturer chooses, there is no loss of efficiency because of incentive problems.

Recall that the manufacturer is able to assemble $x_{1}$ end-products when all suppliers have the low-cost realization (the probability of this happening is $p^{n}$ ) and $x_{2}$ end-products otherwise. The manufacturer's objective function (as of Step 1) is therefore:

$$
\Pi=R p^{n} x_{1}+R\left(1-p^{n}\right) x_{2}-n\left(p c_{1} x_{1}^{2}+(1-p) c_{2} x_{2}^{2}\right) .
$$

\section{Proposition 1: The optimal Team solution is either $a$ :}

i) Separating solution when $\left(p^{n-1}-p^{n}\right) /\left(1-p^{n}\right) \geq c_{1} / c_{2}$ :

$$
x_{1}^{T}=\frac{R p^{n-1}}{2 n c_{1}}, x_{2}^{T}=\frac{R\left(1-p^{n}\right)}{2 n c_{2}(1-p)} .
$$

ii) Pooling solution when $\left(p^{n-1}-p^{n}\right) /\left(1-p^{n}\right)<c_{1} / c_{2}$ :

$$
x_{1}^{T}=x_{2}^{T}=x^{T}=\frac{R}{2 n\left(p c_{1}+(1-p) c_{2}\right)} .
$$

See Appendix A for the proof. In the Separating solution, the manufacturer specifies two yield rates, one for each cost realization. In the Pooling solution the manufacturer specifies only one yield rate, regardless of the suppliers' cost realizations. The advantage of the Pooling solution is that all suppliers provide the same yield rate and the same number of non-defective parts. As a result, the manufacturer does not end up paying for non-defective parts that cannot be used in an end-product. The disadvantage of the Pooling solution is that the manufacturer cannot benefit from the possibility that all of the suppliers are low-cost by having each produce a higher yield, resulting in more endproducts that can be sold at a profit. Notice that the manufacturer chooses either the Pooling or the Separating solution based on the problem parameters.

The Team solution is a trade-off between the two effects discussed above. As the probability of the low-cost realization increases (i.e., $p$ increases), it becomes more likely that the manufacturer is facing all low-cost suppliers. It is, thus, benefitical to fully exploit each of them and therefore, the Separating solution dominates. To see this effect, consider the derivative of the Separating/Pooling threshold with respect to $p$ 


$$
\frac{\partial}{\partial p} \frac{p^{n-1}-p^{n}}{1-p^{n}}=\frac{\left((n-1) p^{n-2}-n p^{n-1}\right)\left(1-p^{n}\right)+n p^{n-1}\left(p^{n-1}-p^{n}\right)}{\left(1-p^{n}\right)^{2}}=p^{n-2} \frac{n(1-p)-1+p^{n}}{\left(1-p^{n}\right)^{2}} \geq 0 .
$$

Because the numerator is decreasing in $p$ with the smallest value being non-negative at $p=1$, the threshold is indeed increasing in $p$.

Further, for any given $p$, as the number of parts increases, the probability of there being at least one high-cost supplier who will provide parts with a lower yield rate increases. This makes it more likely that the "extra parts" supplied by the low-cost suppliers will not be utilized in an end-product, making the Pooling solution more attractive. To verify this, consider the derivative of the Separating/Pooling threshold with respect to $n$

$$
\frac{\partial}{\partial n} \frac{p^{n-1}-p^{n}}{1-p^{n}}=\frac{\left(p^{n-1}-p^{n}\right)\left(1-p^{n}\right) \ln p+\left(p^{n-1}-p^{n}\right) p^{n} \ln p}{\left(1-p^{n}\right)^{2}}=\left(p^{n-1}-p^{n}\right) /\left(1-p^{n}\right)^{2} \ln p<0
$$

Thus, the threshold is decreasing in $n$. Likewise, as the ratio of costs, $c_{1} / c_{2}$, increases (i.e., the difference in costs becomes smaller), there is less reason to treat suppliers of different types differently, and the Pooling solution becomes more advantageous.

Finally, note also that optimal yields are decreasing in $n$ due to the weakest link problem This is because as $n$ increases the manufacturer has to pay for more parts, the cost of the product increases, and therefore, it is less valuable to assemble a non-defective end-product. ${ }^{15}$

In the next two sections, we study the yields induced by three different types of contracts. Unless otherwise noted, we will assume that the exogenous parameter values $\left(c_{1}, c_{2}, p, n\right)$ are such that the optimal yields given those contracts are strictly less than 1. For instance, in terms of the Team solution this would imply that $R p^{n-1} /\left(2 n c_{1}\right) \leq 1$ and $R \leq\left(2 n\left(p c_{1}+(1-p) c_{2}\right)\right)$. This is consistent with the philosophy of Juran who, unlike Deming, believes that less than perfect quality is optimal (Juran 1992).

\footnotetext{
${ }^{15}$ We could have normalized for this effect of adding suppliers by assuming that the revenue function was $n \mathrm{R}$. However, the incremental effect arising from the underlying contracting problem would remain the same.
} 


\section{The manufacturer tests parts before assembly}

In the remainder of this paper, we retain the assumption of asymmetric information but drop the assumption that each supplier will choose her yield as instructed but the manufacturer. Instead, each supplier will choose whichever yield rate maximizes her expected utility given her cost realization and the contract offered to her by the manufacturer. Thus, in choosing between the suppliers' contracts, the manufacturer must now consider their incentive effects on the suppliers' behavior.

In this section, we examine two different types of contracts between the manufacturer and the suppliers for the situation in which the manufacturer (costlessly and perfectly) tests all of the incoming parts. We first examine the AQL contract in which the manufacturer specifies alternative acceptable yield rates, each with a different payment. We then examine the $\mathrm{Q}$ - Pricing contract in which the manufacturer accepts any yield rate from each supplier and offers each a constant payment $T$ per nondefective part. In both of these situations we assume that each supplier can only observe the outcome of the testing of her own parts. As a result, each supplier can only be compensated based on the results of her own tested parts, not on the results of other suppliers' tested parts. Further, in both contracts, after testing only good parts will be used to assemble the end-products, resulting in $\min \left(x_{1 \bullet}, x_{2 \bullet}, \ldots, x_{n \bullet}\right)$ end-products assembled, where $x_{i \bullet}$ represents the supplier $i$ 's yield given her cost realization.

\section{a. AQL Contract}

With an Acceptable Quality Level contract, the manufacturer specifies the yields for which he will compensate the suppliers while all other yields are not acceptable and not rewarded. Given the incentive problems that underlie our supply chain model, the contract which maximizes the manufacturer's expected profit is, without loss of generality, an AQL contract. ${ }^{16}$ Thus, without loss of generality (see Kreps 1990), the manufacturer can restrict himself to contracts which: 1) specify two acceptable yield rates, one for suppliers with a low-cost realization and one for suppliers with a highcost realization; 2) induce each supplier to choose the yield rate consistent with her cost realization and anticipated by the manufacturer; and 3) assure each supplier at least her outside opportunity cost

\footnotetext{
${ }^{16}$ This result is based on the Revelation Principle. See Myerson (1979) and Harris and Townsend (1981).
} 
regardless of her cost realization. Denote by $P$ the set of all permutations of $n$ cost indices with each taking values 1 or 2 . Further, denote by $P(k, n)$ the subset of these permutations such that there are $k$ low-cost realizations of $n$ suppliers. Given this definition, the manufacturer's problem is

$$
\max _{x_{i j}, T_{i j}} \sum_{k=0}^{n}\left\{R p^{k}(1-p)^{n-k} \sum_{P(k, n) \in P} \min \left(x_{1 \bullet}, x_{2}, \ldots, x_{n \bullet}\right)\right\}-p \sum_{i=1}^{n} T_{i 1}-(1-p) \sum_{i=1}^{n} T_{i 2}
$$

subject to

$$
\begin{array}{ll}
T_{i 1}-c_{1} x_{i 1}^{2} \geq 0 & \forall i \\
T_{i 2}-c_{2} x_{i 2}^{2} \geq 0 & \forall i \\
T_{i 1}-c_{1} x_{i 1}^{2} \geq T_{i 2}-c_{1} x_{i 2}^{2} & \forall i \\
T_{i 2}-c_{2} x_{i 2}^{2} \geq T_{i 1}-c_{2} x_{i 1}^{2} & \forall i
\end{array}
$$

The objective function takes the expectation over all possible permutations of low-cost and high-cost suppliers. Constraints (LL1) and (LL2) are supplier $i$ 's Limited Liability constraints and assure that, regardless of supplier $i$ 's cost realization, she will earn at least her outside opportunity cost and hence will implement the contract negotiated in Step 1. Constraints (IC1) and (IC2) are the Incentive Compatibility constraints which assure that the supplier behavior anticipated by the manufacturer is in each supplier's best interest. That is, if supplier $i$ is a low-cost supplier she will prefer to choose $x_{i 1}$ rather than $x_{i 2}$ (IC1) and vice versa if she is a high-cost supplier (IC2). The objective function above is different from that for the Team solution because we allow for the possibility that the manufacturer will offer different contracts to otherwise identical suppliers.

Proposition 2: The optimal AQL contract is either a:

i) Separating solution when $p^{n-1} \geq c_{1} / c_{2}$ :

$$
\begin{aligned}
& x_{i 1}=x_{j 1}=x_{1}>x_{i 2}=x_{j 2}=x_{2} \text { and } T_{1}=T_{i 1}=T_{j 1}>T_{i 2}=T_{j 2}=T_{2} \forall i, j \\
& x_{1}=p^{n-1} R / 2 n c_{1}, x_{2}=\left(1-p^{n}\right) R / 2 n\left(c_{2}-p c_{1}\right) \\
& T_{1}=c_{1}\left(\frac{p^{n-1} R}{2 n c_{1}}\right)^{2}+\left(c_{2}-c_{1}\right)\left(\frac{\left(1-p^{n}\right) R}{2 n\left(c_{2}-p c_{1}\right)}\right)^{2}, T_{2}=c_{2}\left(\frac{\left(1-p^{n}\right) R}{2 n\left(c_{2}-p c_{1}\right)}\right)^{2} \\
& \text { The manufacturer's expected profit is } \frac{p^{2 n-1} R^{2}}{4 n c_{1}}+\frac{\left(1-p^{n}\right)^{2} R^{2}}{4 n\left(c_{2}-p c_{1}\right)}
\end{aligned}
$$


The supplier's expected profit is $p\left(c_{2}-c_{1}\right)\left(\frac{\left(1-p^{n}\right) R}{2 n\left(c_{2}-p c_{1}\right)}\right)^{2}$

ii) Pooling solution when $p^{n-1}<c_{1} / c_{2}$ :

$x_{i j}=x$ and $T_{i j}=T \forall i, j$

$x=R / 2 n c_{2}$

$T=R^{2} / 4 n c_{2}$

The manufacturer's expected profit is $R^{2} / 4 n c_{2}$

The supplier's expected profit is $R^{2} p\left(c_{2}-c_{1}\right) / 4 n^{2} c_{2}^{2}$.

The same logic for the Separating vs. Pooling Team solutions applies in the case of AQL contract. Under both types of AQL solutions, the low-cost supplier earns more than her minimum expected profit while the high-cost supplier earns just her outside opportunity. The amount that the low-cost supplier earns in excess of her outside opportunity wage is referred to as her informational rent. It is the amount that is required to get the low-cost supplier to honestly reveal her cost realization. This is a standard result for such screening problems and follows from the fact that it is more expensive for a high-cost supplier to act as if she were a low-cost supplier than vice versa (see Kreps 1990).

We are interested in studying how the optimal AQL yield rates vary in the exogenous parameters and how that variation is affected by the existence of contracting problems. To isolate the variation in the optimal AQL yields that arises strictly from contracting problems, we restate them in terms of the Team solution yields. Recall that the Team problem has asymmetric information but no incentive problems (each supplier will implement the yield decision rule desired by the manufacturer as long as she cannot improve her expected profit by supplying the parts to another manufacturer. Therefore, any difference in the optimal yields for the Team problem and for the AQL problem is due solely to incentive problems. The difficulty in making this comparison, however, is that both the AQL and Team solutions can result in Separating and Pooling solutions which may occur over different sets of parameters. We therefore begin by comparing the Separating/Pooling thresholds as functions of $n$, i.e., $p^{n-1} v s\left(p^{n-1}-p^{n}\right) /\left(1-p^{n}\right)$. It is straightforward to see that $p^{n-1}>\left(p^{n-1}-p^{n}\right) /\left(1-p^{n}\right)$, and, thus, holding all other parameters fixed, the threshold $n$ for the Separating AQL contract is always lower than for the Separating Team solution. As a result, separation of suppliers is maintained over a larger 
range of problem parameters for the AQL contract. Figure 1 illustrates the comparison between Team solution and $\mathrm{AQL}$ contract.

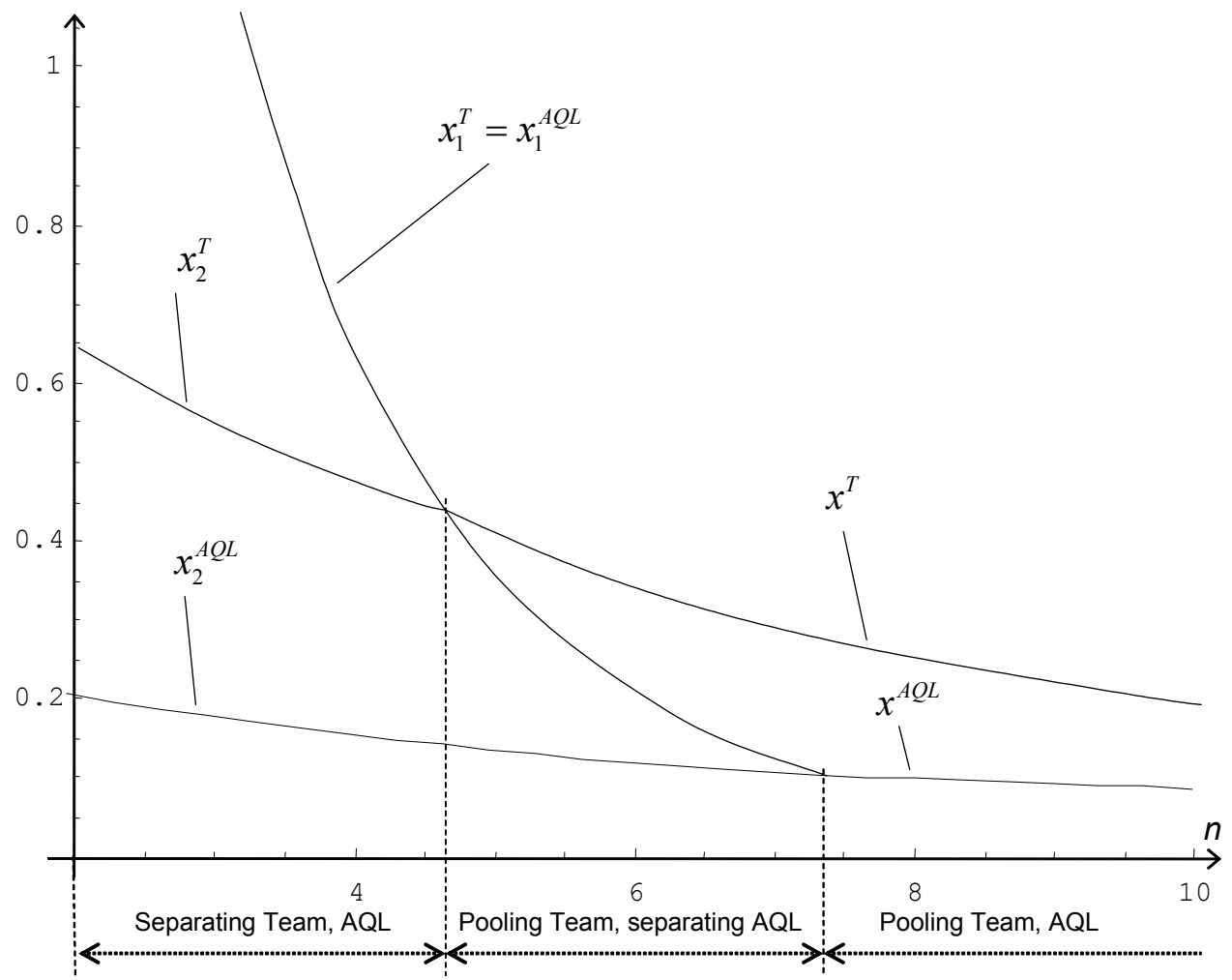

Figure 1. Optimal yield rates with the AQL contract.

As is evident from the picture, three cases have to be considered:

Case 1. $p^{n-1} \geq\left(p^{n-1}-p^{n}\right) /\left(1-p^{n}\right) \geq c_{1} / c_{2}$. Both the AQL and Team solutions are Separating. We can rewrite the $\mathrm{AQL}$ yields as:

$$
x_{1}=x_{1}^{T}, x_{2}=x_{2}^{T} \frac{c_{2}(1-p)}{\left(c_{2}-p c_{1}\right)} .
$$

In this case, production by low-cost suppliers is not distorted away from that achievable without incentive problems. The high-cost suppliers' yield, on the other hand, is less than the Team solution because of incentive problems. ${ }^{17}$ Interestingly, the relative distortion in $x_{2}$ is not affected by the number of parts, $n$. Hence, within the range of parameters in Case 1, changing the number of parts that make up the final end-product does not affect the incentive costs associated with the AQL contract.

\footnotetext{
${ }^{17}$ In most screening problems the low-cost supplier's action choice is First-Best, while the high-cost supplier's action choice is distorted from First-Best (see Kreps 1990). The First-Best solution is not the relevant comparison for our problem, because even without incentive problems, we still have asymmetric information. Our Case 1 result is therefore similar to the standard result in screening problems where the comparison is now to the Team solution
} 
However, $p$ does affect the distortion in $x_{2}$, relative to the Team solution. As $p$ increases, the multiple for $x_{2}^{T}$ decreases thus aggravating the production distortion for high-cost suppliers. The intuition is that an increase in $p$ implies a lower probability of a high-cost supplier. Therefore, the value of the high-cost supplier's production is less and the manufacturer can reduce the low-cost supplier's informational rent $\left(T_{1}=c_{1}\left(x_{1}\right)^{2}+\left(c_{2}-c_{1}\right)\left(x_{2}\right)^{2}\right)$ by reducing the high-cost supplier's yield rate, $x_{2}$.

Case 2. $p^{n-1} \geq c_{1} / c_{2} \geq\left(p^{n-1}-p^{n}\right) /\left(1-p^{n}\right)$. The AQL solution is Separating while the Team solution is Pooling. We can rewrite the AQL yields as:

$$
x_{1}=x^{T} \frac{\left(p c_{1}+(1-p) c_{2}\right) p^{n-1}}{c_{1}}, x_{2}=x^{T} \frac{\left(p c_{1}+(1-p) c_{2}\right)\left(1-p^{n}\right)}{c_{2}-p c_{1}} .
$$

In this case both the low-cost and high-cost suppliers' yield rates are distorted below their respective Team optimal yield rates (see also Figure 1). ${ }^{18}$ Now the number of parts that make up the end-product does have an incentive effect in the sense that the more parts that make up the end-product, the greater (less) the distortion induced in the low-cost (high-cost) supplier's yield, relative to the Team solution. Intuitively, as $n$ increases the chance of facing low-cost realizations from all suppliers diminishes. Thus, for the Separating AQL solution it becomes optimal to reduce the difference in the low and highcost yields and, thereby, reduce the low-cost supplier's information rent.

Case 3. $c_{1} / c_{2} \geq p^{n-1} \geq\left(p^{n-1}-p^{n}\right) /\left(1-p^{n}\right)$. Both AQL and Team solutions are pooling so that $x=x^{T}\left(p c_{1}+(1-p) c_{2}\right) / c_{2}$.

Note that the AQL optimal yield is distorted below the Team yield. Like Case 1 and unlike Case 2, the impact of the incentive problem in Case 3 does not depend on the number of parts, because the effect of $n$ in both the AQL and Team yields is the same. As $p$ increases, the AQL yield decreases relative to the Team solution thus amplifying the effect of the incentive problem. The reason is that an increase in $p$ results in the manufacturer paying informational rent to more suppliers. Hence, to decrease the total rent paid, the manufacturer further reduces the AQL yield.

\footnotetext{
${ }^{18}$ Note that in Case 2 the actions of all suppliers, in particular the low-cost suppliers, are distorted. This is different than the standard result for screening contract in which the low-cost supplier's yield is always First-Best. The reason for this difference is that in the typical screening problem there are no externalities among the suppliers. Therefore, each supplier's First-Best action depends solely on her own private information, whereas in our model, there are externalities.
} 
To summarize, with the AQL contract the inefficiency introduced by incentive problems manifests itself in "over-separation" of supplier yields (see Figure 1) in two senses. First, the optimal yields for the low and high-cost suppliers are always farther apart under the AQL contract than under the Team solution. Second, the Separating AQL solution is implemented for a larger set of problem parameters than for the Team solution. Interestingly, the number of parts that make up the end-product does not always affect the distortion in yields resulting in incentive problems (Cases 1 and 3).

\section{b. Q - Pricing Contract}

In the $\mathrm{Q}$ - Pricing contract we retain all of the same assumptions as for the AQL contract, except now the manufacturer pays each supplier $T$ for the proportion of her parts that are good, $x_{i}$. As noted earlier, the AQL contract will maximize the manufacturer's expected utility given the underlying incentive problems. Therefore, using the $\mathrm{Q}$ - Pricing contract will, in general, result in a lower expected utility for the manufacturer. As a result, any distortion in the optimal yields relative to the Team solution will be due to the underlying incentive problems and the use of a sub-optimal contract. In spite of this, we study the $\mathrm{Q}$ - Pricing contract because it is simpler to design than the AQL contract and is used in both practice and in the literature (e.g., Reyniers and Tapiero 1995).

Under the Q - Pricing contract, each supplier's optimization problem for cost realization $c_{i}$ is (dropping the supplier subscript)

$$
\max _{x_{i}} T x_{i}-c_{i} x_{i}^{2} .
$$

Given this arrangement, the optimal yield rate for the supplier with cost realization $c_{i}$ is

$$
x_{i}=T / 2 c_{i} .
$$

Each supplier's expected profit (as of Step 1) is:

$$
\pi=p\left(T x_{1}-c_{1} x_{1}^{2}\right)+(1-p)\left(T x_{2}-c_{2} x_{2}^{2}\right)=\frac{T^{2}}{4}\left(\frac{c_{1}+p\left(c_{2}-c_{1}\right)}{c_{1} c_{2}}\right) .
$$

The manufacturer's objective function (as of Step 1) is: 


$$
\Pi=R\left(p^{n} x_{1}+\left(1-p^{n}\right) x_{2}\right)-n T\left(\frac{p x_{1}}{2 c_{1}}+\frac{(1-p) x_{2}}{2 c_{2}}\right)
$$

Substituting (1) into the manufacturer's objective function and optimizing with respect to $T$ gives the optimal payment for each good part: $T=\frac{R}{2 n} \frac{c_{1}+p^{n}\left(c_{2}-c_{1}\right)}{c_{1}+p\left(c_{2}-c_{1}\right)}$. Substituting that back into (1) gives the optimal yield rate for a supplier with a cost realization $c_{j}$ :

$$
x_{j}=T^{*} / 2 c_{j}=\frac{R}{4 n c_{j}} \frac{c_{1}+p^{n}\left(c_{2}-c_{1}\right)}{c_{1}+p\left(c_{2}-c_{1}\right)} .
$$

The manufacturer's expected profit (as of Step 1) is

$$
\Pi=\frac{R^{2}}{8 n c_{1} c_{2}} \frac{\left(c_{1}+p^{n}\left(c_{2}-c_{1}\right)\right)^{2}}{c_{1}+p\left(c_{2}-c_{1}\right)}
$$

and each supplier's expected profit (as of Step 1) is

$$
\pi=\frac{R^{2}}{16 n^{2} c_{1} c_{2}} \frac{\left(c_{1}+p^{n}\left(c_{2}-c_{1}\right)\right)^{2}}{c_{1}+p\left(c_{2}-c_{1}\right)} .
$$

Notice that with the $\mathrm{Q}$ - Pricing contract, if a supplier has cost realization $c_{i}$, her profit, conditional on that realization, is $T x_{i}-c_{i} x_{i}^{2}=T^{2} / 4 c_{i}>0$. This exceeds her outside opportunity cost of zero and therefore, the supplier will agree to implement the optimal Q - Pricing contract.

As with the AQL contract, to isolate the variation in the optimal yields that arises strictly from contracting problems, we restate the optimal Q-Pricing yields in terms of the Team solution yields. Because the Team solution may be Pooling or Separating, two cases have to be considered.

Case 1. $\left(p^{n-1}-p^{n}\right) /\left(1-p^{n}\right) \geq c_{1} / c_{2}$. The Team solution is Separating. In this case the optimal yield rates under Q - Pricing can be restated as:

$$
\begin{aligned}
& x_{1}=x_{1}^{T}\left(\frac{1}{2}\right)\left[\frac{1}{p^{n-1}} \frac{c_{1}+p^{n}\left(c_{2}-c_{1}\right)}{c_{1}+p\left(c_{2}-c_{1}\right)}\right], \\
& x_{2}=x_{2}^{T}\left(\frac{1}{2}\right)\left[\frac{1-p}{1-p^{n}} \frac{c_{1}+p^{n}\left(c_{2}-c_{1}\right)}{c_{1}+p\left(c_{2}-c_{1}\right)}\right] .
\end{aligned}
$$


As with the AQL contract, under Q - Pricing, any supplier with a low (high) cost realization will be induced to choose a yield which is a multiple of the corresponding Team solution yield, where the multiple arises solely from the existence of incentive problems. It can be verified that the multiple for low-cost suppliers can be greater or less than one while the multiple for high-cost suppliers is always less than one. Therefore, incentive problems may cause the low-cost suppliers to produce either more or less than the Team amount while the incentive problems always cause the high-cost supplier to always produce less than the Team amount.

The multiples in (6) represent the production distortions arising from the underlying incentive problem. For $n=1$, we get $x_{j}=0.5 x_{j}^{T}$ so that both of the Q-Pricing yields are distorted below the Team yields. As the number of parts increases, $x_{1}$ increases relative to $x_{1}^{T}$ since the multiple is increasing in $n:$

$$
\frac{\partial}{\partial n}\left(\frac{1}{2}\right)\left[\frac{1}{p^{n-1}} \frac{c_{1}+p^{n}\left(c_{2}-c_{1}\right)}{c_{1}+p\left(c_{2}-c_{1}\right)}\right]=\left(\frac{1}{2}\right)\left[-\frac{c_{1} \ln p}{p^{n-1}\left(c_{1}(1-p)+c_{2} p\right)}\right]>0 .
$$

Thus, an increase in $n$ initially mitigates the production distortion associated with the low-cost supplier. However, note that at the maximum $n$ consistent with Case 1, the optimal Q-Pricing yield for the low-cost supplier is

$$
\left.x_{1}\right|_{\left(p^{n-1}-p^{n}\right) /\left(1-p^{n}\right)=c_{1} / c_{2}}=x_{1}^{T}\left(\frac{1}{2}\right)\left[\frac{c_{2}}{c_{1}+p\left(c_{2}-c_{1}\right)}\right],
$$

which may be greater than the Team yield. Unlike the case for the low-cost supplier, the multiple for $x_{2}$ always decreases in $n$. Therefore, the production distortion associated with the high-cost suppliers caused by the incentive problem is further aggravated by adding parts.

Case 2. $\left(p^{n-1}-p^{n}\right) /\left(1-p^{n}\right)<c_{1} / c_{2}$. The Team solution is Pooling. In this case the optimal yield rates under Q-Pricing are:

$$
\begin{aligned}
& x_{1}=x^{T}\left(\frac{1}{2}\right)\left[\frac{c_{1}+p^{n}\left(c_{2}-c_{1}\right)}{c_{1}+p\left(c_{2}-c_{1}\right)} \frac{\left(p c_{1}+(1-p) c_{2}\right)}{c_{1}}\right], \\
& x_{2}=x^{T}\left(\frac{1}{2}\right)\left[\frac{c_{1}+p^{n}\left(c_{2}-c_{1}\right)}{c_{1}+p\left(c_{2}-c_{1}\right)} \frac{\left(p c_{1}+(1-p) c_{2}\right)}{c_{2}}\right] .
\end{aligned}
$$

The multiple due to incentive problems is decreasing in $n$ for both low-cost and high-cost suppliers. For high-cost suppliers the multiple is always less than 1 and therefore, increasing $n$ increases the 
production distortion caused by the underlying incentive problems. As in Case 1, whether an increase in $n$ mitigates of exacerbates the production distortion caused by the incentive problems depends on the parameter values.

To understand the effect of the number of parts on the production distortion under Q-Pricing notice that an increase in $n$ increases the probability that there is at least one high-cost supplier and therefore the excess amounts produced by low-cost suppliers are wasted. The manufacturer would like to reduce $x_{1}$ and increase $x_{2}$. However, the only lever of control is his choice of $T$ which will only allow him to increase or decrease both yields.

\section{Group Warranty}

Suppose now that the manufacturer assembles the end-product without first testing the individual parts. ${ }^{19}$ Under the Group Warranty contract, if an assembled product fails, the suppliers are collectively and equally responsible for the failure. That is, each supplier is paid $T$ for each assembled end-product that does not fail (the assembled product only functions if all parts are non-defective), and zero if the assembled end-product does not function. ${ }^{20}$ Even though each supplier's compensation depends upon the actions of the other suppliers, we assume that suppliers cannot either help each other or coordinate their efforts. We also assume that suppliers can costlessly verify when the end-product actually failed.

Contrary to the other two contracts we considered, under Group Warranty each supplier's payoff is not independent of decisions by other suppliers. Consider, for example, supplier 1. For each of the two cost realizations, supplier 1 knows only the probability distribution of the other suppliers' cost realizations. She also knows that when her parts are assembled with the other suppliers' parts, the yield rate of a good assembled end-product is the product of the yield rates of all suppliers' parts. Hence, supplier 1 is interested in the expected yield of all suppliers other than herself. Denote this

\footnotetext{
${ }^{19}$ The manufacturer might decide not to test incoming parts if assembly is cheap or testing parts is costly or it is only possible to determine if parts are good by testing the end-product (e.g., testing is destructive as is true with airbags) or it is impossible (or too costly) to discover which supplier's part was faulty if an assembled product fails.

20 An alternative is to share Group Warranty costs between the manufacturer and suppliers. However, because the manufacturer does not affect product quality in our model, sharing Group Warranty costs does not introduce any additional interesting tradeoffs - it merely transfers money from the manufacturer to suppliers. It can be easily shown that if the manufacturer is in charge of the contract design (as is the case in our model), he would allocate full Group Warranty costs to suppliers. Hence, for the reminder of the paper we assume that Group Warranty costs are fully born by suppliers.
} 
expectation by $X$. Dropping the supplier subscript, supplier 1 solves the following problem for each of her cost realizations, $c_{j}$

$$
\max _{x_{j}} x_{j} X T-c_{j} x_{j}^{2}
$$

From the first-order conditions, the optimal yield for a supplier with cost realization $c_{j}$ is

$$
x_{j}=\min \left(X T / 2 c_{j}, 1\right) \text {. }
$$

Assuming symmetry, $X$ can be written as $X=\left(p x_{1}+(1-p) x_{2}\right)^{n-1}$ because the suppliers' cost realizations are independent. One can observe that $X$ is an increasing function of the other suppliers' chosen yield rates. Hence, (8) indicates that the higher (lower) the other $n$-1 suppliers' expected yield rates the higher (lower) will be supplier 1 's chosen yield rates and profits. The same is true for all suppliers so that the suppliers' chosen yield rates are strategic complements. Thus, with a Group Warranty arrangement, for any given $T$ each supplier is better off if the other suppliers increase their yield rates. Notice that this effect does not arise in the cases in which there is testing before assembly, because each supplier's reward is then independent of the other suppliers' yields. The game that arises among suppliers is similar to "coordination games" (see Cachon and Camerer 1996) in which the payoff to every player is determined by the one player whose strategy is the "weakest link". The difference, however, is that in our problem there is a principal (manufacturer) who can change the outcome of the game by setting $T$. The manufacturer's profit is

$$
\Pi=(R-n T)\left(p x_{1}+(1-p) x_{2}\right)^{n} .
$$

Proposition 3: The solution under Group Warranty is:

i) for $n \geq 3$, one of the following three solutions (the one resulting in the highest manufacturer's profit) is an equilibrium:

a. $x_{1}=x_{2}=0$ with the manufacturer's profit of $\Pi=0$.

b. $x_{1}=1, x_{2}=c_{1} / c_{2}$ with the manufacturer's profit of

$$
\Pi=R\left(p+(1-p) c_{1} / c_{2}\right)^{n}-2 n c_{1}\left(p+(1-p) c_{1} / c_{2}\right) .
$$

c. $x_{1}=x_{2}=1$ with the manufacturer's profit of $\Pi=R-2 n c_{2}$. 
ii) for $n=2$, in addition to the above three options, the following equilibrium must be considered $^{21}$

$$
x_{1}=1, x_{2}=\frac{R(1-p)-2 c_{2}}{4 c_{2}-R(1-p)} \frac{p}{(1-p)}
$$

as long as $c_{1} / c_{2}<x_{2}<1$ with the manufacturer's profit

$$
\Pi=\frac{p^{2} 4 c_{2}^{2}}{(1-p)\left(4 c_{2}-R(1-p)\right)} .
$$

Because the suppliers' yield choices are strategic complements, we might expect that in equilibrium all suppliers will gravitate toward the boundary solution in which all of them will either select the maximum or minimum possible quality. If $R$ is relatively small, the manufacturer cannot make positive profits under Group Warranty and hence will refuse to contract with suppliers inducing equilibrium yields of zero (case a). As $R$ increases, at some point the manufacturer begins making positive profits and it becomes optimal for him to offer positive compensation $T>0$ to the suppliers. Because the suppliers' decisions are complements, it is relatively easy to induce them to select high yields. As a result, a small increase in $T$ leads to a large increase in both high-cost and low-cost suppliers' yields followed by an increase in the manufacturer's profit. Note from (8) that if the solutions for both yields are interior, then $x_{1} / x_{2}=c_{2} / c_{1}$ so that as the manufacturer increases $T$, yields increase while preserving this proportion until $x_{1}=1, x_{2}=c_{1} / c_{2}$ which are the maximum yields such that the solution is still interior. At this point, further increase in $T$ will only increase yields of the high-cost suppliers $\left(x_{2}\right)$ so that the return to increasing $T$ is less. This is why for some problem parameters the manufacturer will choose equilibrium $\mathrm{b}$ for $n \geq 3$. However, it is also possible that, for some problem parameters, a further increase in $T$ is warranted. If that is the case, it will be optimal to raise $T$ until $x_{1}=x_{2}=1$ ( case $\mathrm{c}$ for $n \geq 3$ ).

If, however, $n=2$, it is possible to have a solution with $c_{1} / c_{2}<x_{2}<1$ because of the cost function. To see this in more detail, let $x_{1}=1$ and recall that $T=2 c_{2} x_{2} / X$ so that manufacturer's objective function becomes

\footnotetext{
${ }^{21}$ The number of equilibria is actually larger for $n=2$ (see the proof) but we use Pareto-dominance to eliminate most of them.
} 


$$
\Pi=\left(R-\frac{2 n c_{2} x_{2}}{X}\right)\left(p+(1-p) x_{2}\right)^{n}=R\left(p+(1-p) x_{2}\right)^{n}-2 n c_{2} x_{2}\left(p+(1-p) x_{2}\right) .
$$

Notice that the cost term is quadratic in $x_{2}$ while the revenue term has power $n$. If $n=2$, there are problem parameters such that the objective function is concave quadratic and a unique interior maximum exists.

Because up to 4 different equilibria can arise, we will introduce assumptions which eliminate all but the most interesting equilibrium. The solution with $x_{1}=x_{2}=0$ is uninteresting so we will assume that problem parameters are such that the manufacturer makes positive profits under Group Warranty. Furthermore, the solution with $x_{1}=x_{2}=1$ is also uninteresting. We will eliminate it for the time being as well (see Proposition 7 where this solution is discussed) by assuming that $R<2 n c_{2}$ which implies that the manufacturer's profit is negative when $x_{1}=x_{2}=1$. This is a rather mild parametric assumption: note that the optimal yield under the Pooling AQL solution is $R /\left(2 n c_{2}\right)$ so we are simply assuming that this yield is less than 1 . Finally, for $n=2$, there is only a very narrow range of problem parameters such that an interior solution characterized by (9) actually arises. ${ }^{22}$ Hence, from now on we will work with the equilibrium in which $x_{1}=1, x_{2}=c_{1} / c_{2}$, the only non-trivial equilibrium arising for a wide range of problem parameters (which we also confirmed through extensive numerical studies).

We now analyze the distortions in the yields under Group Warranty arising from the underlying incentive problems. Because $x_{1}=1$ under Group Warranty, it is clear that the low-cost suppliers' yields are distorted above the Team solution and that the distortion increases in $n$. On the other hand, under Group Warranty the high-cost suppliers may have a yield that is below or above the corresponding Team solution yield. As $n$ increases, the Group Warranty yield for high-cost suppliers further increases relative to the Team solution yield so that, depending on problem parameters, three situations may arise with respect to $x_{2}$ : an increase in $n$ may increase the distortion, may decrease the distortion, or it may initially decrease but then increase the distortion. These distortions arise because

${ }^{22}$ The following four conditions must hold simultaneously: $2 c_{2}<R(1-p)<4 c_{2}, \frac{c_{1}}{c_{2}}<\frac{R(1-p)-2 c_{2}}{4 c_{2}-R(1-p)} \frac{p}{(1-p)}<1$. 
the Team yields are decreasing in the number of suppliers while the Group Warranty yields are constant.

In equilibrium, the payment to suppliers is:

$$
T=\frac{2 c_{1}}{x_{1}^{n-2}\left(p+(1-p) c_{1} / c_{2}\right)^{n-1}}=\frac{2 c_{1}}{\left(p+(1-p) c_{1} / c_{2}\right)^{n-1}},
$$

each supplier's profit is

$$
\pi=p\left(x_{1} X T-c_{1} x_{1}^{2}\right)+(1-p)\left(x_{2} X T-c_{2} x_{2}^{2}\right)=\left(X T-c_{1}\right)\left(p+\frac{c_{1}}{c_{2}}(1-p)\right)=\frac{c_{1}}{c_{2}}\left(c_{1}+p\left(c_{2}-c_{1}\right)\right),
$$

and the manufacturer's profit is

$$
\Pi=R\left(\frac{c_{1}+p\left(c_{2}-c_{1}\right)}{c_{2}}\right)^{n}-\frac{2 n c_{1}}{c_{2}}\left(c_{1}+p\left(c_{2}-c_{1}\right)\right) .
$$

Note that each supplier's expected profit does not depend on the number of suppliers. However, the manufacturer's expected profit is decreasing in the number of suppliers. This is the result of the optimal quality being independent of the number of suppliers.

\section{Comparison among contracts}

In this section we compare the three contractual arrangements studied previously. Tables 1 and 2 in Appendix B summarize the optimal solutions for the AQL, Q-Pricing and Group Warranty contracts. We proceed by comparing relative benefits of these contracts for the manufacturer, suppliers, and the supply chain as a whole.

Proposition 4: The manufacturer's preference ordering is: $A Q L \geq Q$-Pricing $\geq$ Group Warranty.

It has been argued in the practitioner literature (see Windham 1995) that manufacturers should favor contracts rewarding suppliers for higher quality over contracts that establish acceptable quality levels. In our context this would imply that manufacturers should prefer Q-Pricing contracts to AQL contracts. In contrast, we find the opposite is true. The reason is that any optimal Q-Pricing contract is a feasible AQL contract (see the Proof of Proposition 4). 
The manufacturer is worse off under the Group Warranty contract than under the other two contracts. Apparently, the Group Warranty contract affords the manufacturer so little control regardless of the parameters $\left(x_{1}=1, x_{2}=c_{1} / c_{2}\right)$ that it is less preferred by him than the other contracts. At first glance, we should be able to use the Informativeness Condition (Holmstrom 1979) to argue that the manufacturer prefers the AQL contract to the Group Warranty contract. Clearly the former allows the manufacturer to base the suppliers' contracts on strictly more informative variables. However, the argument doesn't hold here because going from parts testing to end-product testing with an end-product that is characterized by the "weakest link" actually changes not just the set of contractible variables but also the objective function from the minimum over all yields to the product of all the yields,

The results of Proposition 4 are appealing from a practical point of view. While the AQL contract may not be as easy to state and enforce as the Q - Pricing contract, for a large set of parameters (i.e., $p^{n-1}<c_{1} / c_{2}$ ) the AQL contract will yield a Pooling solution in which the manufacturer need only specify one acceptable yield rate and one payment. Furthermore, the Pooling solution under the AQL contract coincides with what is widely observed in industrial practice (see Windham 1995). We next analyze the relative attractiveness of the contracts to the suppliers.

\section{Proposition 5: For suppliers}

a) Group Warranty $\geq Q$-Pricing and Group Warranty $\geq A Q L$.

b) The ranking of $Q$-Pricing vs $A Q L$ is ambiguous. However, if $A Q L \geq Q$-Pricing for a given $n^{*}$, then it is also true for any $n>n$ *.

Intuition suggests that suppliers should not find the Group Warranty contract attractive because it makes them solely responsible for external product failures - even failures caused by other suppliers. However, the suppliers prefer a Group Warranty to the other contracts because the former induces complementarities among suppliers that result in higher yield rates. Table 2 indicates that for both Group Warranty and Q - Pricing the suppliers' expected profit is increasing in induced yield rates. The suppliers prefer Group Warranty to AQL because under the former the suppliers are able to earn rents for both cost realizations while under the latter they are able to earn rents only for the high-cost realization. 
The suppliers may prefer Q-Pricing to AQL contracts, or vice versa. To see why, recall that under AQL suppliers do not make any profits when $p \rightarrow 0$ or when $p \rightarrow 1$ because in these cases the manufacturer can extract all informational rents from suppliers. On the other hand, under Q-Pricing suppliers earn non-negative rents regardless of their cost realizations. Hence, suppliers prefer the QPricing contract for low and high values of $p$. However, we have conducted numerical experiments and have found that when the AQL contract yields a Pooling solution, the suppliers prefer it to the QPricing contract. This is intuitive because in the Pooling solution the manufacturer does not differentiate between low-cost and high-cost suppliers thus extracting less informational rent from these suppliers and leaving them with higher profits. The three graphs below illustrate how the preference ordering between Q-Pricing (dashed line) and AQL (solid line) can change.
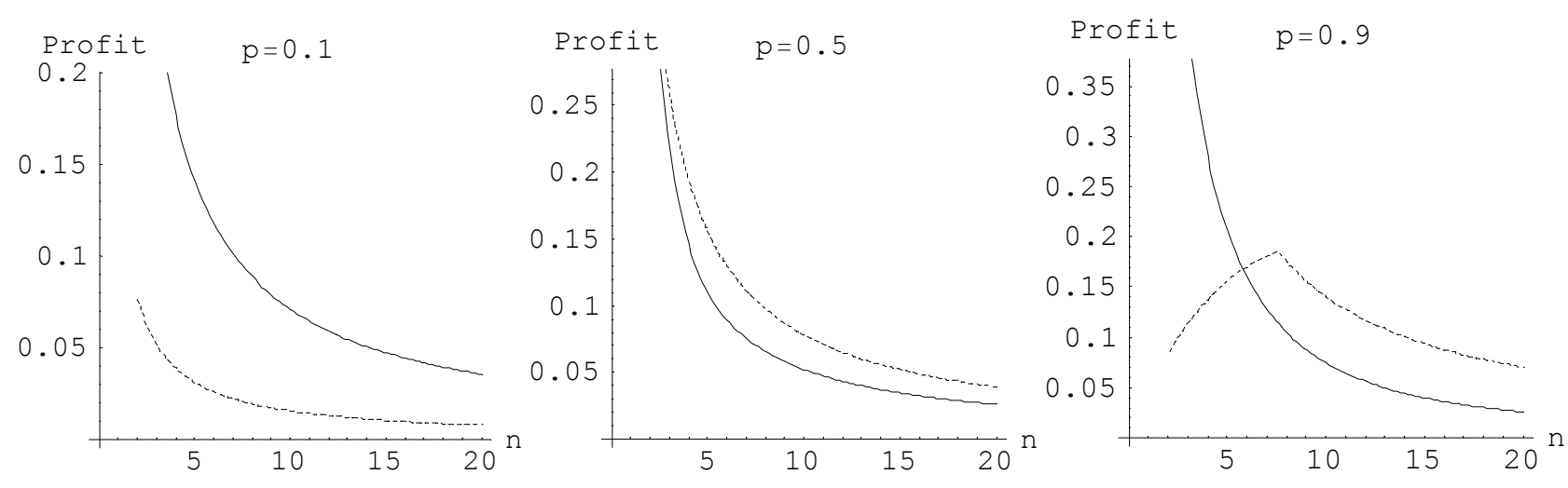

Figure 2. Suppliers' profits under low, moderate and high $p\left(c_{1}=1, c_{2}=2, R=5\right)$.

Finally, consider the efficiency of the overall supply chain with different contracts.

Proposition 6: For the supply chain

a) Total expected profits are greater under pooling AQL than Q-Pricing.

b) Total expected profits are greater under pooling AQL than Group Warranty.

Although part a) is stated only for the pooling AQL contract, we believe that it holds for the separating contract as well. The same is not always true for part b). Figure 3 below indicates that there exist cases in which the supply chain is better off with Group Warranty (dotted line) contracts than with either Q - Pricing (solid line) or AQL contracts that result in a Separating solution (dashed lines). 

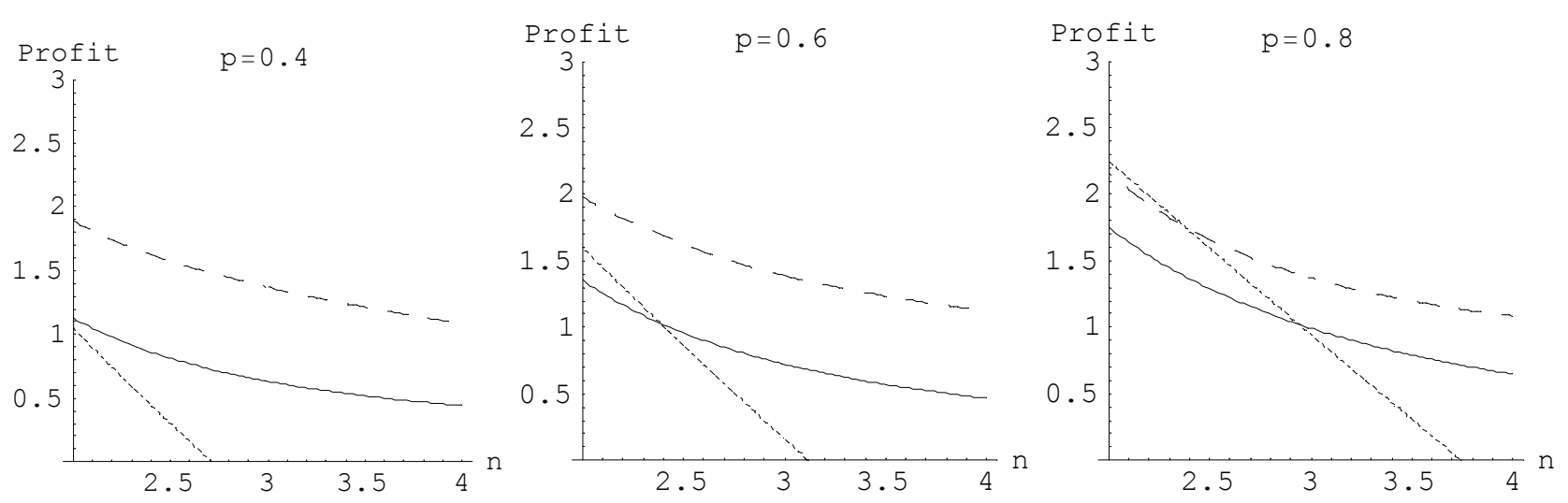

Figure 3. Supply chain profits under low, moderate and high $p\left(c_{1}=1, c_{2}=2, R=5\right)$.

The graphs indicate that Group Warranty is most preferred by the supply chain only when there are few parts and the probability of a low-cost realization is high. This combination of problem parameters leads to small distortion of Group Warranty yields from the Team solution and hence better contract performance. For a majority of problem parameters we find that the AQL contract does very well, especially when a large number of parts is involved. This is the direct consequence of the result that the manufacturer always prefers AQL and also that suppliers typically prefer AQL to Q-Pricing for large $n$. Another way to explain this result is by recalling that, as $n$ increases, distortion of yields under Pooling AQL contract is not amplified while under the other two contracts it is amplified for one or both yields.

To this point, we have assumed that less than perfect quality is optimal. If it is optimal to induce $x_{1}=x_{2}=1$, what would be the most efficient contractual relationship for doing so? Proposition 7 below addresses that question.

Proposition 7: Assuming that the manufacturer wants to induce perfect quality regardless of the cost-of-yield realizations, then:

a) The supply chain profits are the same under all three contracts

b) Q-Pricing and Group Warranty are Pareto equivalent

c) The manufacturer strictly prefers AQL to both Q-Pricing and Group Warranty.

The supply chain profits are the same under all three contracts because all three are inducing perfect quality, and the cost of doing so is irrelevant to supply chain profits. The cost of doing so just affects the manufacturer's and suppliers' shares of the supply chain profits. Result b) follows from the fact that, with each supplier choosing perfect quality, there are no externalities among them. Finally, 
the manufacturer strictly prefers AQL to the other contracts because in order to induce perfect quality, the payment under AQL is $T=c_{2}$ while under Q-Pricing and Group Warranty it is $T=2 c_{2}$.

\section{Discussion}

In this paper we have analyzed the issue of structuring contracts for multiple outsourced parts in the presence of externalities among suppliers in the form of the "weakest-link". We modeled three contracts, compared their advantages and disadvantages to supply chain members and analyzed the underlying incentive problems under each by comparing the yield rates to the Team solution that does not have incentive problems. With a minor exception, under all three contracts the yield rates are distorted from the Team solution but the nature of the distortions differ among the contracts.

In the $\mathrm{Q}$ - Pricing and AQL contracts the optimal yield rates decrease as the number of parts increase. This result arises because of the weakest link property. Therefore, among those products having the weakest link property, we would expect to see a negative relationship between the number of parts/subassemblies and the cost of failure, if those contracts were in place. Of course, while we have treated the weakest link property as a dichotomous variable, in practice it is a continuous variable. Hence, we would expect the strength of this negative relationship to vary in the "weakness" of the link.

Our results either prove or strongly suggest that the AQL contract works well for both the manufacturer and suppliers when the number of parts is large. This outcome is supported by our finding that, somewhat surprisingly; over a large range of problem parameters the incentive problems are unaffected when parts are added under AQL contract (as opposed to the other two contracts). This is because in these regions adding suppliers has the same effect on the Team solution as on the AQL solution. Further, we found that when the number of parts is small the AQL contract specifies several different acceptable levels of quality, while when the number of parts is large, there is only one acceptable level of quality. One way of interpreting this result is that the band of acceptable quality should narrow as the number of parts increases. It would be interesting to see if this relationship holds for different products that are subject to the weakest link property.

One of our interesting results was that the Group Warranty contract may be preferred by the supply chain, but only when there are few parts. We interpret this result as being consistent with the 
observed relationship between Japanese car manufacturers and their first - tier suppliers. In that relationship, the manufacturer does very little testing of incoming parts, works closely with the few first - tier suppliers providing large sub-assemblies, and implicitly promises to share the benefits of collaboration. This relationship is similar to our Group Warranty in that there is little testing of incoming parts and an aligning of the suppliers' interests on the outcome of the end-product rather than their specific parts.

Our results rely on several assumptions. First, we assumed throughout that the testing of incoming parts is costless. The reason for doing so is that the relative cost of parts testing vs. product testing is unclear. In practice these cost can be substantial and different for parts testing vs. endproduct testing. In addition, the external failure of the final product may lead to the loss of customer goodwill which would be borne by the manufacturer making the Group Warranty contract even less attractive to the manufacturer than our results reflect. Second, we assumed that the results of the testing and the choice of yield rates were non-stochastic. Given that the manufacturer and suppliers were all risk-neutral, adding a random component to either testing or the choice of yield rates should not qualitatively change the results.

A third major assumption was symmetry of parts. Without this assumption, the complexity of the model greatly increases making it difficult to analyze the effect of adding parts. Another simplification was to assume that each part is only provided by one supplier. However, multi-sourcing can have benefits that our present model does not capture. This issue is analyzed in Baiman and Netessine (2004). Finally, our analysis was based on a production function which we characterized as having the weakest link property, which we interpreted as the end-product failing if any one part failed. The qualitative results would continue to hold if we examined a setting in which the end-product would fail if any fixed number of components failed.

There are several possible extensions to this line of research. Analyzing the case in which suppliers differ in their ex ante cost characteristics would be an interesting extension. This would lead to different contracts being offered to the different suppliers. However, this would greatly complicate the analysis of the AQL contracts. Second, it would be interesting to see when and if the manufacturer would choose to implement a combination of internal and external testing, rather than each in isolation, as we have assumed. 
It would also be useful to undertake empirical studies in industries which manufacture complex products, such as airlines and automobiles, where a poor quality part can cause the product to malfunction. What type of testing and inspection systems have been established to deal with this weakest link problem? Are firms in these industries utilizing variations of the AQL and Group Warranty systems or some other procedures? Are there special long-term relationships between suppliers and manufacturers that minimize the chances of such failures occurring? Answers to these questions will enable us to understand more fully the challenges firms face when dealing with supply procurement where the end-products exhibits a weakest link property.

\section{References}

Alchian, A. and H. Demsetz. 1972. Production, information costs and economic organization. American Economic Review, Vol.62, 777-795.

Baiman, S., P. Fischer and M.V. Rajan. 2000. Information, contracting, and quality costs. Management Science, Vol.46, No.6, 776-789.

Baiman, S., P. Fischer and M.V. Rajan. 2001. Performance measurement and design in supply chains. Management Science, Vol.47, No.1, 173-188.

Baiman, S. and S. Netessine. 2004. An Incentive Effect of Multiple Sourcing in Supply Chains. Working Paper, University of Pennsylvania.

Baiman, S. and M.V. Rajan. 2002. Incentive issues in inter-firm relationships. Accounting, Organizations and Society, Vol. 27, April, 213-238.

Balachandran K.R. and S. Radhakrishnan. 2003. Quality implications of warranties in a supply chain. Working Paper, New York University.

Bossert, J. L. 1994. Supplier management handbook. ASQ Quality Press.

Cachon, G.P. 2002. Supply chain coordination with contracts. Forthcoming in the Handbook of Operations Management, S. Graves and T. de Kok, editors.

Cachon, G.P. and C.F. Camerer. 1996. Loss-avoidance and forward induction in experimental coordination games. The Quarterly Journal of Economics, Vol. 111, No.1, 165-194.

Demski, J. S. and D. E. M. Sappington. 1984. Optimal incentive contracts with multiple agents. Journal of Economic Theory, Vol. 33, 152 - 171. 
Harris, M., C.H. Kriebel and A. Raviv. 1982. Asymmetric information, incentives and intrafirm resource allocation. Management Science, Vol. 28, No. 6, 604-620.

Harris M. and R.M. Townsend. 1981. Resource allocation under asymmetric information. Econometrica, Vol.49 (1), 33-64.

Heal, G. and H. Kunreuther. 2003. You only die once: managing discrete interdependent risks. Working Paper, University of Pennsylvania.

Holmstrom, B. 1979. Moral hazard and observability. The Rand Journal of Economics, Vol. 10, Spring, 74-91.

Hopp, W.J. and M.L. Spearman. 2000. Factory Physics. McGraw-Hill, NY.

Itoh, H. 1991. Incentives to help in multi-agent situations. Econometrica, Vol. 59, No. 3, 611-636.

Itoh, H. 1994. Job design, delegation and cooperation: a principal-agent analysis. European Economic Review, Vol. 38, 691-700.

Iyer, A., L. Schwarz and S. Zenios 2002, A principal-agent model for product specification and production. Working paper, Krannert School of Management, Purdue University.

Juran, J.M. 1992. Juran on quality by design: the new steps for planning quality into goods and services. Free Press, New York.

Kreps, D. 1990. A course in microeconomic theory. Princeton University Press, N.J.

Kunreuther, H. and G. Heal. 2003. Interdependent security. The Journal of Risk and Uncertainty, Vol.26:2/3, 231-249.

Lim, W.S. 2001. Producer-supplier contracts with incomplete information. Management Science, Vol.47, No.5, 709-715.

Ma, C. 1988. Unique implementation of incentive contracts with multiple agents. Review of Economic Studies, Vol. 55, No.4, p 555-72.

Marschak, J. and R. Radner. 1972. Economic theory of teams. Yale University Press.

Monteverde K. and D. Teece 1982. Supplier switching costs and vertical integration in the automobile industry. The Bell Journal of Economics, Vol.13, 206-213.

Myerson, R.B. 1979. Incentive compatibility and the bargaining problem. Econometrica, Vol.47, No. $1,61-73$.

Pate-Cornell E. and R. Dillon 2001. Probabilistic risk analysis for the NASA space shuttle: a brief history and current work. Reliability Engineering and System Safety, Vol. 74, 345 - 352. 
Pinedo, M., S. Seshadri and E. Zemel. 2003. The Ford-Firestone Case: Part I. Case study, NYU Stern School of Business.

Pryweller, J. 1999. Lear demands rebates from toolmakers. Crain's Detroit Business, November 8, page 37.

Reyniers, D.J. and C.S. Tapiero. 1995. The delivery and control of quality in supplier-producer contracts. Management Science, Vol.41, No.10, 1581-1589.

Smith, D.C. 1997. Risks and rewards: GM suppliers face tough new rules. Ward's Auto World, 33(6), p. 3.

Ulrich, K. 1995. The role of product architecture in the manufacturing firm. Research Policy, Vol.24, 419-440.

Ulrich, K. and S. Eppinger. 2000. Product design and development. McGraw-Hill, NY.

Windham, J. 1995. Implementing Deming's forth point. Quality Progress, Vol.28, \#12, 43-50. 


\section{Appendix A: Proofs of Propositions}

\section{Proof of Proposition 1 (Team solution).}

Two cases need to be considered.

Case 1. $x_{1}>x_{2}$. After differentiating the objective function, we obtain optimality conditions:

$$
\begin{aligned}
& \frac{\partial \Pi}{\partial x_{1}}=R p^{n}-2 n p c_{1} x_{1}=0, \\
& \frac{\partial \Pi}{\partial x_{2}}=R\left(1-p^{n}\right)-2 n(1-p) c_{2} x_{2}=0
\end{aligned}
$$

from which optimal yields follow. It remains to ensure that $x_{1}>x_{2}$ which leads to the condition for the separating solution. Objective function at optimality is

$$
\Pi=R p^{n} x_{1}+R\left(1-p^{n}\right) x_{2}-n\left(p c_{1} x_{1}^{2}+(1-p) c_{2} x_{2}^{2}\right)=R^{2} \frac{p^{2 n-1}(1-p) c_{2}+\left(1-p^{n}\right)^{2} c_{1}}{4 n c_{1} c_{2}(1-p)}
$$

Case 2. $x_{1}=x_{2}=x$. In this case straightforward differentiation leads to the optimal yield. The objective function at optimality is

$$
\begin{aligned}
\Pi & =R x-n x^{2}\left(p c_{1}+(1-p) c_{2}\right) \\
& =\frac{R^{2}}{2 n\left(p c_{1}+(1-p) c_{2}\right)}-\frac{R^{2} n}{4 n^{2}\left(p c_{1}+(1-p) c_{2}\right)^{2}}\left(p c_{1}+(1-p) c_{2}\right) \\
& =\frac{R^{2}}{2 n\left(p c_{1}+(1-p) c_{2}\right)}-\frac{R^{2}}{4 n\left(p c_{1}+(1-p) c_{2}\right)} \\
& =\frac{R^{2}}{4 n\left(p c_{1}+(1-p) c_{2}\right)}
\end{aligned}
$$

Because as long as $\left(p^{n-1}-p^{n}\right) /\left(1-p^{n}\right) \geq c_{1} / c_{2}$ both solutions are feasible, we need to verify that the separating solution indeed dominates over this range of parameters. The difference in expected profits (the Separating solution - the Pooling solution $)=$

$$
=R^{2} \frac{p^{2 n-1}(1-p) c_{2}+\left(1-p^{n}\right)^{2} c_{1}}{4 n c_{1} c_{2}(1-p)}-\frac{R^{2}}{4 n\left(p c_{1}+(1-p) c_{2}\right)}
$$




$$
\begin{aligned}
& =R^{2} p \frac{p^{2 n-2}(1-p)^{2} c_{2}^{2}-2(1-p) c_{1} c_{2} p^{n-1}\left(1-p^{n}\right)+\left(1-p^{n}\right)^{2} c_{1}^{2}}{4 n c_{1} c_{2}(1-p)\left(p c_{1}+(1-p) c_{2}\right)} \\
& =R^{2} p \frac{\left(p^{n-1}(1-p) c_{2}-\left(1-p^{n}\right) c_{1}\right)^{2}}{4 n c_{1} c_{2}(1-p)\left(p c_{1}+(1-p) c_{2}\right)} \geq 0 .
\end{aligned}
$$

Hence, the manufacturer will choose the separating solution everywhere it is feasible and the pooling solution otherwise.

\section{Proof of Proposition 2 (AQL contract).}

Proposition 2 is proved in a series of steps.

Step 1. Constraints (LL1) cannot bind

Proof: Note that $T_{i 1}-c_{1} x_{i 1}^{2} \geq T_{i 2}-c_{1} x_{i 2}^{2}>T_{i 2}-c_{2} x_{i 2}^{2} \geq 0$ The first inequality follows from (IC1), the second from $c_{1}<c_{2}$ and the third from (LL2).

Step 2. Constraints (LL2) must be binding.

Proof: Assume otherwise and suppose that we start with an optimal solution so that all of the constraints were satisfied and (LL1) is not binding. Reduce both $T_{i 1}$ and $T_{i 2}$ by $\varepsilon$. None of the constraints will be violated by the change and the manufacturer increases his objective function. Step 3. $x_{i 1} \geq x_{i 2} \forall i$.

Proof: From (IC1) and (IC2) $c_{1}\left(x_{i 2}^{2}-x_{i 1}^{2}\right) \geq T_{i 2}-T_{i 1} \geq c_{2}\left(x_{i 2}^{2}-x_{i 1}^{2}\right)$. Given, $c_{2}>c_{1} \Rightarrow x_{i 1} \geq x_{i 2}$.

Step 4. The optimal solution must be of the form $x_{i 1}=x_{j 1}=x_{1} \forall i, j$.

Proof: Assume otherwise, $\exists i, j$ such that $x_{i 1}>x_{j 1}$. Let $\lambda_{i j} i=1, . ., n j=1, . ., 4$ be the Lagrange multiplier for the ith supplier's $j$ th constraint. The First-Order conditions with respect to $x_{i 1}$ are $-2 \lambda_{i 3} c_{1} x_{i 1}+2 \lambda_{i 4} c_{2} x_{i 1}=0 \Rightarrow \lambda_{i 3} c_{1}=\lambda_{i 4} c_{2} \Rightarrow \lambda_{i 3}=\lambda_{i 4}=0$, where the latter implication follows from $c_{2}>c_{1}$. Notice that the FOC with respect to $x_{i 1}$ will not involve terms from the objective function because the objective function is of the form $\min (\bullet)$ and $x_{i 1}>x_{j 1} \geq x_{j 2}$. But if constraints (LL1), (IC1) and (IC2) for supplier $i$ are all not binding, then the manufacturer can reduce $T_{i 1}$ by $\varepsilon>0$, and make the manufacturer better off without violating any of the constraints. Hence we have a contradiction.

Step 5. The optimal solution must be of the form $x_{i 2}=x_{j 2}=x_{2} \forall i, j$.

Proof: 
We begin by showing that if $x_{j 2}$ and $x_{k 2}$ are the two smallest yields in the high-cost state then $x_{j 2}=x_{k 2}$. The reminder of the proof then follows by induction. Assume otherwise, $x_{j 2}>x_{k 2}$. The manufacturer's problem becomes:

$\max _{x_{i j}, T_{i j}}\left(\left[(1-p) x_{k 2}+p(1-p) x_{j 2}+\ldots ..\right] R-p T_{j 1}-p T_{k 1}-(1-p) T_{j 2}-(1-p) T_{k 2}-\ldots\right)$

subject to

$$
\begin{array}{ll}
T_{i 1}-c_{1} x_{i 1}^{2} \geq 0 & \forall i \\
T_{i 2}-c_{2} x_{i 2}^{2} \geq 0 & \forall i \\
T_{i 1}-c_{1} x_{i 1}^{2} \geq T_{i 2}-c_{1} x_{i 2}^{2} & \forall i \\
T_{i 2}-c_{2} x_{i 2}^{2} \geq T_{i 1}-c_{2} x_{i 1}^{2} & \forall i
\end{array}
$$

Note that the objective function explicitly includes all the terms involving suppliers $j$ and $k$. Let $\lambda_{i j} i=1, . ., n j=1, . ., 4$ be the Lagrange multiplier for the $i$ th supplier's $j$ th constraint. The First-Order conditions with respect to $T_{k 1}$ are $-p+\lambda_{k 3}-\lambda_{k 4}=0$. Assume that $x_{\mathrm{k} 1}>x_{k 2}$. If both (IC1) and (IC2) were binding then $c_{1}\left(x_{i 2}^{2}-x_{i 1}^{2}\right)=T_{i 2}-T_{i 1}=c_{2}\left(x_{i 2}^{2}-x_{i 1}^{2}\right) \Rightarrow x_{i 2}=x_{i 1}$, which is a contradiction. Hence $\lambda_{k 4}=0$ and $\lambda_{k 3}>0$. Alternatively, assume that $x_{\mathrm{k} 1}=x_{k 2}$. In this case the two IC constraints are identical and we can drop one, say (IC2), and hence, again, $\lambda_{k 4}=0$ and $\lambda_{k 3}>0$. Using the fact that constraints (LL2) and (IC1) are binding for suppliers $j$ and $k$ we can now use the constraints to solve for the optimal payments to suppliers $j$ and $k$, substitute them into the objective function and find the following optimal yields: $x_{k 2}=(1-p) / 2\left(c_{2}-p c_{1}\right), x_{j 2}=p(1-p) / 2\left(c_{2}-p c_{1}\right)$. But this implies that $x_{k 2}>x_{j 2}$ which contradicts our original hypothesis. The remainder of the proof follows by Induction on the next highest yield in the high-cost state.

At this point we are left with two different possible optimal solutions:

$$
\text { a). Pooling }=x_{i j}=x \forall i, j
$$

and

b). Separating $x_{11}=x_{21}=x_{31}=\ldots . .=x_{n 1}=x_{1}>x_{12}=x_{22}=x_{32}=\ldots . .=x_{n 2}=x_{2}$

Step 6. Pooling $x_{i j}=x \forall i, j$ 
With Pooling and the symmetry among the agents, $T_{i j}=T$ and the (IC) constraints can be dropped.

We can use the binding (LL2) constraint for each supplier to solve for $\mathrm{T}$ in terms of $x$. We then substitute that into the objective function which results in an unconstrained maximization in $x$. The resulting solution is: $x=R / 2 n c_{2}$ and $T=c_{2}\left(R / 2 n c_{2}\right)^{2}$ from the binding (LL2) constraint.

The manufacturer's expected profit is $\frac{R^{2}}{4 n c_{2}}$

Each supplier's expected compensation is $c_{2}\left(\frac{R^{2}}{4 n^{2} c_{2}^{2}}\right)=\frac{R^{2}}{4 n^{2} c_{2}}$

Each agent's expected profit is $\frac{R^{2}}{4 n^{2} c_{2}}-\left(p c_{1}+(1-p) c_{2}\right) \frac{R^{2}}{4 n^{2} c_{2}^{2}}=\frac{R^{2}}{4 n^{2} c_{2}^{2}} p\left(c_{2}-c_{1}\right)$

Finally the total supply chain profit is $\frac{R^{2}}{4 n c_{2}}+\frac{R^{2}}{4 n c_{2}^{2}} p\left(c_{2}-c_{1}\right)=\frac{R^{2}}{4 n c_{2}^{2}}\left(c_{2}+p\left(c_{2}-c_{1}\right)\right)$.

Step 7. Separating $x_{11}=x_{21}=x_{31}=\ldots . .=x_{n 1}=x_{1}>x_{12}=x_{22}=x_{32}=\ldots . .=x_{n 2}=x_{2}$

With Separation and the symmetry among the agents, $T_{i j}=T_{k j}=T_{j} j=1,2$ and $\forall i, k$. Recall that the optimal solution is such that for each supplier the (LL2) and (IC1) constraints are binding. This allows us to solve for $T_{1}$ and $T_{2}$ in terms of $x_{1}$ and $x_{2}$. Substituting these into the objective function results in an unconstrained optimization in $x_{1}$ and $x_{2}$. The optimal yield rates are: $x_{1}=p^{n-1} R / 2 n c_{1}$ and $x_{2}=\left(1-p^{n}\right) R / 2 n\left(c_{2}-p c_{1}\right)$. Notice that consistency requires that $x_{1}>x_{2} \Rightarrow p^{n-1} c_{2}>c_{1}$. Hence the Separating solution can only hold when $p^{n-1} c_{2}>c_{1}$.

Using the binding (LL2) and (IC1) constraints to solve for the compensations results in:

$T_{1}=c_{1}\left(\frac{p^{n-1} R}{2 n c_{1}}\right)^{2}+\left(c_{2}-c_{1}\right)\left(\frac{\left(1-p^{n}\right) R}{2 n\left(c_{2}-p c_{1}\right)}\right)^{2}$ and $T_{2}=c_{2}\left(\frac{\left(1-p^{n}\right) R}{2 n\left(c_{2}-p c_{1}\right)}\right)^{2}$.

The manufacturer's expected profit is $\frac{p^{2 n-1} R^{2}}{4 n c_{1}}+\frac{\left(1-p^{n}\right)^{2} R^{2}}{4 n\left(c_{2}-p c_{1}\right)}$

Each supplier's expected profit is 


$$
\begin{aligned}
& (1-p) c_{2}\left(\frac{\left(1-p^{n}\right) R}{2 n\left(c_{2}-p c_{1}\right)}\right)^{2}+p\left[c_{1}\left(\left(\frac{p^{n-1} R}{2 n c_{1}}\right)^{2}-\left(\frac{\left(1-p^{n}\right) R}{2 n\left(c_{2}-p c_{1}\right)}\right)^{2}\right)+c_{2}\left(\frac{\left(1-p^{n}\right) R}{2 n\left(c_{2}-p c_{1}\right)}\right)^{2}\right] \\
& -p c_{1}\left(\frac{p^{n-1} R}{2 n c_{1}}\right)^{2}-(1-p) c_{2}\left(\frac{\left(1-p^{n}\right) R}{2 n\left(c_{2}-p c_{1}\right)}\right)^{2} \\
& =p\left(c_{2}-c_{1}\right)\left(\frac{\left(1-p^{n}\right) R}{2 n\left(c_{2}-p c_{1}\right)}\right)^{2}
\end{aligned}
$$

Finally, the supply chain profit is $\frac{p^{2 n-1} R^{2}}{4 n c_{1}}+\frac{\left(1-p^{n}\right)^{2} R^{2}}{4 n\left(c_{2}-p c_{1}\right)}+p\left(c_{2}-c_{1}\right)\left(\frac{\left(1-p^{n}\right)^{2} R^{2}}{4 n\left(c_{2}-p c_{1}\right)^{2}}\right)$.

Step 8. Comparison of Pooling and Separating Equilibria

Only the Pooling solution can hold when $p^{n-1}<c_{1} / c_{2}$, while both are feasible when $p^{n-1} \geq c_{1} / c_{2}$. To determine which the manufacturer will choose (recall that it is the manufacturer who is offering the contract) we compare his expected profit under both. The manufacturer's expected profit under (the Separating solution - the Pooling solution) $=$

$$
\left(\frac{R^{2}\left(1-p^{n}\right)^{2}}{4 n\left(c_{2}-p c_{1}\right)}\right)+\left[\frac{R^{2} p^{2 n-1}}{4 n c_{1}}\right]-\left(\frac{R^{2}}{4 n c_{2}}\right)=\left(\frac{R^{2} p}{4 n c_{1} c_{2}\left(c_{2}-p c_{1}\right)}\right)\left[p^{n-1} c_{2}-c_{1}\right]^{2}>0
$$

Hence, the manufacturer will choose the Separating solution when $p^{n-1} \geq c_{1} / c_{2}$ and the Pooling solution otherwise.

\section{Proof of Proposition 3 (Group Warranty).}

Case $i$ ), $n \geq 3$. From (8), when $x_{1}<1$, then $x_{2}=x_{1} c_{2} / c_{1}$. In addition, when $x_{1}=1$, it is possible that $x_{2}=x_{1} c_{2} / c_{1}$. Therefore, we start by assuming $x_{2}=x_{1} c_{2} / c_{1}$ and hence we can write

$$
\begin{gathered}
x_{1}=\frac{X T}{2 c_{1}}=\frac{\left(p x_{1}+(1-p) x_{2}\right)^{n-1} T}{2 c_{1}}=\frac{x_{1}^{n-1}\left(p+(1-p) c_{1} / c_{2}\right)^{n-1} T}{2 c_{1}}=\left(\frac{2 c_{1}}{\left(p+(1-p) c_{1} / c_{2}\right)^{n-1} T}\right)^{\frac{1}{n-2}} . \\
\Rightarrow T=\frac{2 c_{1}}{x_{1}^{n-2}\left(p+(1-p) c_{1} / c_{2}\right)^{n-1}} .
\end{gathered}
$$

The expression for T can be substituted into the manufacturer's objective function so that the manufacturer effectively sets $x_{1}$ rather than $T$. The manufacturer's expected profit is 


$$
\begin{aligned}
\Pi^{*} & =(R-n T)\left(p x_{1}+(1-p) x_{2}\right)^{n}=(R-n T)\left(p+(1-p) c_{1} / c_{2}\right)^{n} x_{1}^{n} \\
& =\left(R x_{1}^{n}-\frac{2 c_{1} n x_{1}^{2}}{\left(p+(1-p) c_{1} / c_{2}\right)^{n-1}}\right)\left(p+(1-p) c_{1} / c_{2}\right)^{n} .
\end{aligned}
$$

The first and second derivatives are:

$$
\begin{gathered}
\frac{\partial \Pi}{\partial x_{1}}=\left(R n x_{1}^{n-1}-\frac{4 c_{1} n x_{1}}{\left(p+(1-p) c_{1} / c_{2}\right)^{n-1}}\right)\left(p+(1-p) c_{1} / c_{2}\right)^{n}, \\
\frac{\partial^{2} \Pi}{\partial x_{1}^{2}}=\left(\operatorname{Rn}(n-1) x_{1}^{n-2}-\frac{4 c_{1} n}{\left(p+(1-p) c_{1} / c_{2}\right)^{n-1}}\right)\left(p+(1-p) c_{1} / c_{2}\right)^{n} .
\end{gathered}
$$

From (11) we find that $x_{1}^{n-2}=4 c_{1} /\left(R\left(p+(1-p) c_{1} / c_{2}\right)^{n-1}\right)$ which we further substitute into the second derivative:

$$
\begin{aligned}
\left.\frac{\partial^{2} \Pi}{\partial x_{1}^{2}}\right|_{\frac{\partial \Pi}{\partial x_{1}}=0} & =\left(R n(n-1) \frac{4 c_{1}}{R\left(p+(1-p) c_{1} / c_{2}\right)^{n-1}}-\frac{4 c_{1} n}{\left(p+(1-p) c_{1} / c_{2}\right)^{n-1}}\right)\left(p+(1-p) c_{1} / c_{2}\right)^{n} \\
& =((n-1)-1) 4 n c_{1}\left(p+(1-p) c_{1} / c_{2}\right) \geq 0 .
\end{aligned}
$$

The second derivative is strictly positive (FOC characterizes a global minimum). Hence, the global maximum will be on the boundary and either $\left(x_{1}=1, x_{2}=c_{1} / c_{2}\right)$ or $\left(x_{1}=x_{2}=0\right)$. We have therefore established parts $\mathrm{a}$ and $\mathrm{b}$.

To establish part $\mathrm{c}$, assume that $x_{1}=1$ but drop the previous assumption that $x_{2}=x_{1} c_{2} / c_{1}$, and allow $c_{1} / c_{2} \leq x_{2} \leq 1$. Assuming an interior solution for $x_{2}$, from $(8), x_{2}=\left(p+(1-p) x_{2}\right)^{n-1} T /\left(2 c_{2}\right)$. The manufacturer's profit is

$$
\begin{aligned}
\Pi & =(R-n T)\left(p+(1-p) x_{2}\right)^{n} \\
& =R\left(p+(1-p) x_{2}\right)^{n}-2 n c_{2} x_{2}\left(p+(1-p) x_{2}\right) .
\end{aligned}
$$

The first and second derivatives are

$$
\begin{aligned}
\frac{\partial \Pi}{\partial x_{2}}= & R n(1-p)\left(p+(1-p) x_{2}\right)^{n-1}-2 n c_{2} p-4 n c_{2}(1-p) x_{2} \\
\frac{\partial^{2} \Pi}{\partial x_{2}^{2}} & =R n(n-1)(1-p)^{2}\left(p+(1-p) x_{2}\right)^{n-2}-4 n c_{2}(1-p) \\
& =n(1-p)\left(R(n-1)(1-p)\left(p+(1-p) x_{2}\right)^{n-2}-4 c_{2}\right) .
\end{aligned}
$$


From (12) we find that $R(1-p)\left(p+(1-p) x_{2}\right)^{n-2}=\frac{2 c_{2} p+4 c_{2}(1-p) x_{2}}{\left(p+(1-p) x_{2}\right)}$ and substitute this into the second derivative:

$$
\begin{aligned}
\left.\frac{\partial^{2} \Pi}{\partial x_{2}^{2}}\right|_{\frac{\partial \Pi}{\partial x_{2}}=0} & =n(1-p)\left((n-1) \frac{2 c_{2} p+4 c_{2}(1-p) x_{2}}{p+(1-p) x_{2}}-4 c_{2}\right) \\
& =2 c_{2} n(1-p)\left(\frac{p(n-3)+2(1-p) x_{2}(n-2)}{p+(1-p) x_{2}}\right) .
\end{aligned}
$$

The second derivative is strictly positive so that the global maximum is on one of the boundaries, $x_{2}=c_{1} / c_{2}$ or $x_{2}=1$. This establishes case $\mathrm{c}$.

Case $i$ ), $n=2$. To establish parts a and $\mathrm{b}$ we repeat the proof with the assumption that $x_{1}=x_{2} c_{1} / c_{2}$ but notice from (10) that $T=2 c_{1} /\left(p+(1-p) c_{1} / c_{2}\right)$. This implies that both first-order conditions simplify to $x_{1} / x_{2}=c_{2} / c_{1}$ so that any yields satisfying this equality will be an equilibrium. However, it is straightforward to verify that the largest of these equilibria is Pareto-optimal so that $x_{1}=1$ and $x_{2}=c_{1} / c_{2}$ which establishes $\mathrm{b}$. Notice that in this case $T$ does not depend upon the yields so effectively the manufacturer either pays suppliers a fixed $T$ according to (10) and induces the above equilibrium or pays nothing and induces $x_{1}=x_{2}=0$ which establishes a.

To establish $\mathrm{c}$ and $\mathrm{d}$, we repeat the proof based on the assumption that $x_{1}=1$ and $c_{1} / c_{2} \leq x_{2} \leq 1$. Observe from (13) that the second derivative is strictly negative so that there is a unique global maximum for $x_{2}$ found from (12) which is $x_{2}=\frac{R(1-p)-2 c_{2}}{4 c_{2}-R(1-p)} \frac{p}{(1-p)}$, assuming that $c_{1} / c_{2} \leq x_{2}=\frac{R(1-p)-2 c_{2}}{4 c_{2}-R(1-p)} \frac{p}{(1-p)} \leq 1$ which establishes d. Otherwise, the solution will be on either boundary, $x_{2}=c_{1} / c_{2}$ or $x_{2}=1$ thereby establishing $\mathrm{b}$ and $\mathrm{c}$.

\section{Proof of Proposition 4 (Manufacturer's profit comparison).}

Part I. Q-Pricing $\geq$ Group Warranty. We need to show

$$
\frac{R^{2}}{8 n c_{1} c_{2}} \frac{\left(c_{1}+p^{n}\left(c_{2}-c_{1}\right)\right)^{2}}{c_{1}+p\left(c_{2}-c_{1}\right)}-R\left(\frac{c_{1}+p\left(c_{2}-c_{1}\right)}{c_{2}}\right)^{n}+\frac{2 n c_{1}}{c_{2}}\left(c_{1}+p\left(c_{2}-c_{1}\right)\right) \geq 0 .
$$


Observe that this expression is quadratic in $R$ and that the coefficient of $R^{2}$ is positive. Therefore if we can show that the determinant of this quadratic function is non-positive, this will imply that the parabola representing the left-hand side of this inequality lies at or above $x$-axis and hence (14) always holds. Hence, we need to show

$$
\begin{aligned}
& \left(\frac{c_{1}+p\left(c_{2}-c_{1}\right)}{c_{2}}\right)^{2 n} \leq 4 \frac{2 n c_{1}}{c_{2}}\left(c_{1}+p\left(c_{2}-c_{1}\right)\right) \frac{1}{8 n c_{1} c_{2}} \frac{\left(c_{1}+p^{n}\left(c_{2}-c_{1}\right)\right)^{2}}{c_{1}+p\left(c_{2}-c_{1}\right)} \\
& \left(\frac{c_{1}+p\left(c_{2}-c_{1}\right)}{c_{2}}\right)^{2 n} \leq\left(\frac{c_{1}+p^{n}\left(c_{2}-c_{1}\right)}{c_{2}}\right)^{2}, \\
& (y+p(1-y))^{n} \leq y+p^{n}(1-y), \text { where } y=c_{1} / c_{2} .
\end{aligned}
$$

Observe that the inequality holds as equality at the extremes $(y=0$ and $y=1)$. Hence, it remains to show that the inequality holds at every intermediate point. The first and second derivatives of the left-hand side (LHS) and the right-hand side (RHS) are:

$$
\begin{aligned}
& \frac{\partial L H S}{\partial y}=n(1-p)(y+p(1-y))^{n-1} \geq 0, \frac{\partial^{2} L H S}{\partial y^{2}}=n(n-1)(1-p)^{2}(y+p(1-y))^{n-2} \geq 0, \\
& \frac{\partial R H S}{\partial y}=1-p^{n} \geq 0, \frac{\partial^{2} R H S}{\partial y^{2}}=0 .
\end{aligned}
$$

The LHS and the RHS are both increasing functions but the RHS is linear while the LHS is convex. It follows that LHS $\leq$ RHS for any $0<y<1$ and any $n$.

Part II. AQL $\geq Q$-Pricing. This follows from the fact that the optimal Q - Pricing contract is a feasible AQL contract. To see this, let $\left(T, x_{1}, x_{2}\right)$ be the optimal Q - Pricing contract. This is Pareto equivalent to an AQL contract of the following form $\left(\left(T_{1}=T x_{1}, x_{1}\right),\left(T_{1}=T x_{2}, x_{2}\right)\right)$, which, by definition, satisfies both LL and IC constraints. Therefore it is a feasible AQL contract.

\section{Proof of Proposition 5 (Suppliers' profit comparison).}

Part a). Group Warranty $\geq Q$-Pricing. We need to show

$$
\frac{R^{2}}{16 n c_{1} c_{2}} \frac{\left(c_{1}+p^{n}\left(c_{2}-c_{1}\right)\right)^{2}}{c_{1}+p\left(c_{2}-c_{1}\right)} \leq \frac{n c_{1}}{c_{2}}\left(c_{1}+p\left(c_{2}-c_{1}\right)\right)
$$

Observe that the LHS can be expressed in terms of the optimal yield rate under Q-Pricing (see Table 2 in Appendix B) allowing us to reexpress the above as: 


$$
n\left(c_{1}+p\left(c_{2}-c_{1}\right)\right) x_{1} x_{2} \leq n c_{1}\left(c_{1}+p\left(c_{2}-c_{1}\right)\right) / c_{2}
$$

Further, recall that $x_{1} / x_{2}=c_{2} / c_{1}$ so we get $x_{1}^{2} c_{1} / c_{2} \leq c_{1} / c_{2}$ because the yield cannot exceed 1 , and the result follows.

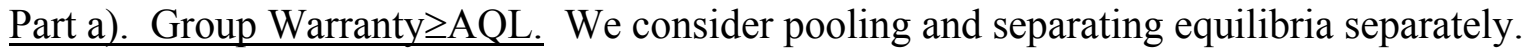

a.1) For the pooling AQL we need to show

$$
\frac{n c_{1}}{c_{2}}\left(c_{1}+p\left(c_{2}-c_{1}\right)\right) \geq \frac{R^{2}}{4 n c_{2}^{2}} p\left(c_{2}-c_{1}\right) .
$$

Clearly, the inequality holds for large $n$ because the LHS is increasing and the RHS is decreasing in $n$. Hence, it suffices to show that (15) holds for the smallest $n$, e.g., $n=1$. Further, recall that we just demonstrated that Group Warranty $\geq$ Q-Pricing for all parameters including $n=1$. Hence, it suffices to show that Q-Pricing $\geq$ AQL for $n=1$ in order to establish that Group Warranty $\geq A Q L$ for all parameters. Thus, we need to demonstrate

$$
\begin{aligned}
& \frac{R^{2}}{16 n c_{1} c_{2}} \frac{\left(c_{1}+p^{n}\left(c_{2}-c_{1}\right)\right)^{2}}{c_{1}+p\left(c_{2}-c_{1}\right)}>\frac{R^{2}}{4 n c_{2}^{2}} p\left(c_{2}-c_{1}\right), \\
& \frac{\left(c_{1}+p\left(c_{2}-c_{1}\right)\right)}{4 c_{1}}>\frac{p\left(c_{2}-c_{1}\right)}{c_{2}}, \\
& \frac{c_{1} c_{2}}{\left(4 c_{1}-c_{2}\right)\left(c_{2}-c_{1}\right)}>p .
\end{aligned}
$$

It suffices to demonstrate that the LHS is greater than 1:

$$
\begin{aligned}
& \frac{c_{1} c_{2}}{\left(4 c_{1}-c_{2}\right)\left(c_{2}-c_{1}\right)}>1>p, \\
& 0>-\left(2 c_{1}-c_{2}\right)^{2} .
\end{aligned}
$$

a.2) Next we will show that Group Warranty $\geq$ Separating AQL. To this point we have demonstrated that Group Warranty $\geq$ pooling AQL without using the fact that $p^{n-1}<c_{1} / c_{2}$. Separating AQL and pooling AQL are defined over different ranges of parameters. However, as we show next, the suppliers' profit under pooling AQL is more than suppliers' profit under separating AQL even over the 
range of parameters for which the separating AQL holds. Thus, the proof will be completed if we show that for $p^{n-1}>c_{1} / c_{2}$, we have

$$
\frac{R^{2}}{4 n c_{2}^{2}} p\left(c_{2}-c_{1}\right)>\frac{p\left(c_{2}-c_{1}\right)}{n}\left(\frac{\left(1-p^{n}\right) R}{2\left(c_{2}-p c_{1}\right)}\right)^{2}
$$

To see this, recall that both profits have the same expression $n p\left(c_{2}-c_{1}\right) x_{2}^{2}$ when written in terms of the their respective optimal yield rates (see Table 2). Therefore, it suffices to show that $x_{2}$ is higher under pooling AQL than under separating AQL:

$$
\frac{R}{2 n c_{2}} \geq \frac{R\left(1-p^{n}\right)}{2 n\left(c_{2}-p c_{1}\right)} \Leftrightarrow \frac{c_{1}}{c_{2}} \leq p^{n-1}
$$

To summarize, we have already shown that Group Warranty>pooling AQL for any problem parameters and we have also shown that pooling AQL>separating AQL when $p^{n-1}>c_{1} / c_{2}$. Hence, Group Warranty>separating AQL.

Part b). Q-Pricing vs AQL. To establish part b) we need to show that the difference (Q-Pricing-AQL) is monotonically decreasing in $n$ whenever Q-Pricing $>\mathrm{AQL}$. That implies that there exists at most one $n^{*}$ such that the suppliers are indifferent between $\mathrm{Q}-$ Pricing and AQL at $n *$ and strictly prefer AQL for $n>n *$.

b.1) AQL pooling. Consider the expression (Q-Pricing-pooling AQL):

$$
\frac{R^{2}}{16 n c_{1} c_{2}} \frac{\left(c_{1}+p^{n}\left(c_{2}-c_{1}\right)\right)^{2}}{c_{1}+p\left(c_{2}-c_{1}\right)}-\frac{R^{2}}{4 n c_{2}^{2}} p\left(c_{2}-c_{1}\right)=\frac{R^{2}}{4 n}\left(\frac{1}{4 c_{1} c_{2}} \frac{\left(c_{1}+p^{n}\left(c_{2}-c_{1}\right)\right)^{2}}{c_{1}+p\left(c_{2}-c_{1}\right)}-\frac{p\left(c_{2}-c_{1}\right)}{c_{2}^{2}}\right) .
$$

The term in front of the bracket is decreasing in $n$ and positive and the term inside the bracket is decreasing in $n$ so the result follows.

b.2) For Q-Pricing vs separating AQL consider once again the expression (Q-Pricing-separating $\mathrm{AQL)}$ :

$$
\frac{R^{2}}{16 n c_{1} c_{2}} \frac{\left(c_{1}+p^{n}\left(c_{2}-c_{1}\right)\right)^{2}}{c_{1}+p\left(c_{2}-c_{1}\right)}-\frac{p\left(c_{2}-c_{1}\right)}{n}\left(\frac{\left(1-p^{n}\right) R}{2\left(c_{2}-p c_{1}\right)}\right)^{2}=\frac{R^{2}}{4 n}\left(\frac{\left(c_{1}+p^{n}\left(c_{2}-c_{1}\right)\right)^{2}}{4 c_{1} c_{2}\left(c_{1}+p\left(c_{2}-c_{1}\right)\right)}-\frac{p\left(c_{2}-c_{1}\right)\left(1-p^{n}\right)^{2}}{\left(c_{2}-p c_{1}\right)^{2}}\right)
$$

Again, the first term is decreasing positive in $n$ and the second term is decreasing in $n$. 


\section{Proof of Proposition 6 (supply chain profit comparison).}

Part a). AQL Pooling $\geq$ Q-Pricing. We need to prove:

$$
\frac{3 R^{2}}{16 n c_{1} c_{2}} \frac{\left(c_{1}+p^{n}\left(c_{2}-c_{1}\right)\right)^{2}}{c_{1}+p\left(c_{2}-c_{1}\right)} \leq \frac{3 R^{2}}{16 n c_{1} c_{2}} \frac{\left(c_{1}+p \frac{c_{1}}{c_{2}}\left(c_{2}-c_{1}\right)\right)^{2}}{c_{1}+p\left(c_{2}-c_{1}\right)} \leq \frac{R^{2}}{4 n c_{2}^{2}}\left(c_{2}+p\left(c_{2}-c_{1}\right)\right),
$$

where the first inequality follows from $p^{n}<p c_{1} / c_{2}$. Simplify

$$
\begin{aligned}
& \frac{\left(c_{1}+p \frac{c_{1}}{c_{2}}\left(c_{2}-c_{1}\right)\right)^{2}}{c_{1}+p\left(c_{2}-c_{1}\right)} \leq \frac{4 c_{1}}{3 c_{2}}\left(c_{2}+p\left(c_{2}-c_{1}\right)\right), \\
& c_{1}^{2}+2 p \frac{c_{1}^{2}}{c_{2}}\left(c_{2}-c_{1}\right)+p^{2} \frac{c_{1}^{2}}{c_{2}^{2}}\left(c_{2}-c_{1}\right)^{2} \leq \frac{4 c_{1}^{2}}{3}+p \frac{4 c_{1}}{3 c_{2}}\left(c_{2}-c_{1}\right)\left(c_{1}+c_{2}\right)+p^{2} \frac{4 c_{1}}{3 c_{2}}\left(c_{2}-c_{1}\right)^{2},
\end{aligned}
$$

After collecting similar terms we obtain

$$
-p^{2}\left(c_{2}-c_{1}\right)^{2} \frac{c_{1}}{c_{2}}\left(\frac{4}{3}-\frac{c_{1}}{c_{2}}\right)-p\left(c_{2}-c_{1}\right) \frac{2 c_{1}}{3 c_{2}}\left(2 c_{2}-c_{1}\right)-\frac{c_{1}^{2}}{3} \leq 0 .
$$

Each term on the LHS is negative so the inequality always holds.

Part b). AQL pooling $\geq$ Group Warranty.

The difference in total expected profits between AQL pooling and Group Warranty is:

$$
\frac{R^{2}}{4 n c_{2}^{2}}\left(c_{2}+p\left(c_{2}-c_{1}\right)\right)-R\left(\frac{c_{1}+p\left(c_{2}-c_{1}\right)}{c_{2}}\right)^{n}+\frac{n c_{1}}{c_{2}}\left(c_{1}+p\left(c_{2}-c_{1}\right)\right)
$$

which is clearly positive for large enough $\mathrm{n}$. This difference is quadratic in $R$ therefore to prove that the above is always strictly positive all we need to do is prove that there are no real roots in $\mathrm{R}$ or equivalently that the determinant is less than zero - this would imply that the above parabola is always above the $\mathrm{x}$ - axis.

$$
\begin{gathered}
\left(\frac{c_{1}+p\left(c_{2}-c_{1}\right)}{c_{2}}\right)^{2 n}<\frac{c_{1}}{c_{2}^{3}}\left(c_{2}+p\left(c_{2}-c_{1}\right)\right)\left(c_{1}+p\left(c_{2}-c_{1}\right)\right), \\
(y+p(1-y))^{2 n-1}<y(1+p(1-y)), \text { where } y=c_{1} / c_{2} .
\end{gathered}
$$

Recall that under pooling AQL, $p^{n-1}<y$. Observe that the RHS of (16) is linearly increasing in $p$ and the LHS is increasing and convex in $p$. Moreover, for the smallest $p, p=0$ we have $y^{2 n-1}<y$ because $y<1$. Hence, if we can show that the inequality also holds for the largest possible $p^{n-1}=y$ the proof is 
complete because the convex function (LHS) will always be below the linear function (RHS). Notice that the LHS is decreasing in $n$ (since the expression in the brackets is less than 1). So all we need to do is prove this result for the smallest $n, n=2$. To summarize, we only need to prove the inequality for $n=2$ and the maximum $p$ allowed by the constraint $p^{n-1}=p<y$. We substitute the maximum possible $p$ into the initial expression (16)

$$
\begin{aligned}
& (y+y(1-y))^{3}<y(1+y(1-y)), \\
& (y+y(1-y))^{3}<(y+y(1-y))^{2}<y(1+y(1-y)),
\end{aligned}
$$

since the term inside the cube is less than 1 . Further,

$$
\begin{aligned}
& \left(2 y-y^{2}\right)^{2}<y+y^{2}-y^{3}, \\
& y^{3}-3 y^{2}+3 y-1=(y-1)^{3}<0,
\end{aligned}
$$

which always holds for $\mathrm{y}<1$.

\section{Proof of Proposition 7 (perfect quality).}

To establish the results we solve each contract separately.

\section{Part i) Q-Pricing}

With Q-Pricing, the manufacturer has to pay $T=2 c_{2}$ in order to assure that the high-cost supplier will supply perfect quality (see (1)). The low-cost supplier, facing compensation of $T=2 c_{2}$ per nondefective part, would like to produce even higher quality but is constrained to produce no more than perfect quality. This results in the following:

supplier expected profit $=2 c_{2}-\left(p c_{1}+(1-p) c_{2}\right)$, manufacturer expected profit $=R-2 n c_{2}$ supply chain expected profit $=R-2 n c_{2}+2 n c_{2}-n\left(p c_{1}+(1-p) c_{2}\right)=R-n\left(p c_{1}+(1-p) c_{2}\right)$

Part ii) AQL

With AQL, the manufacturer pays the supplier only if parts are perfect and zero otherwise. So the Incentive Compatibility constraints require only that the supplier prefers to supply perfect quality to zero quality, i.e., $T-c_{1} \geq 0$ and $T-c_{2} \geq 0$. Hence $T=c_{2}$. This results in the following:

supplier expected profit $=p\left(c_{2}-c_{1}\right)$, manufacturer expected profit $=R-n c_{2}$,

supply chain expected profit $=R-n c_{2}+n c_{2}-n\left(p c_{1}+(1-p) c_{2}\right)=R-n\left(p c_{1}+(1-p) c_{2}\right)$.

\section{Part iii) Group Warranty}


Given that the suppliers are induced to produce perfect quality regardless of their types, $X_{-i}=1 \forall i$. Hence, (1) and (8) are equal and Group Warranty and Q-Pricing settings are identical. All the results immediately follow by comparing the above profit expressions. 


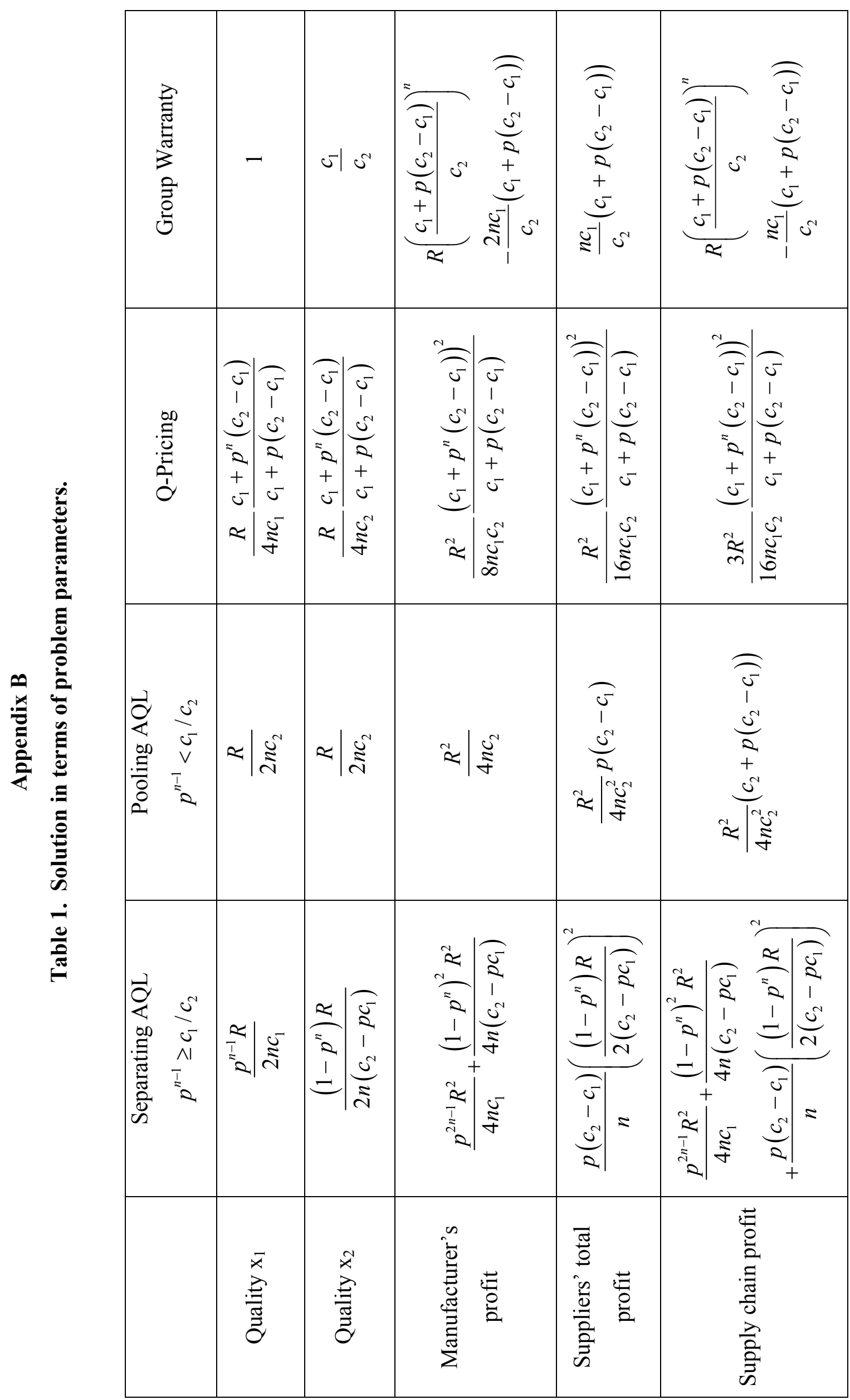




\begin{tabular}{|c|c|c|c|c|c|}
\hline 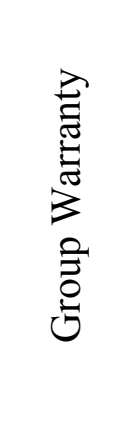 & - & $v \mid v^{v}$ & 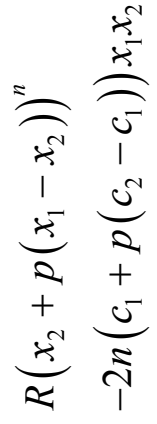 & 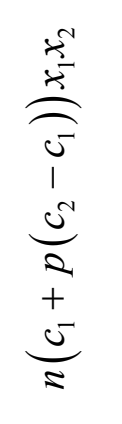 & 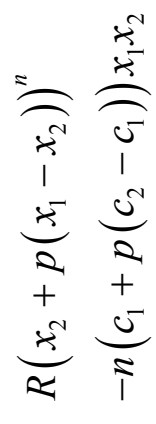 \\
\hline 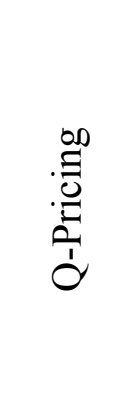 & 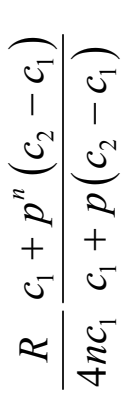 & 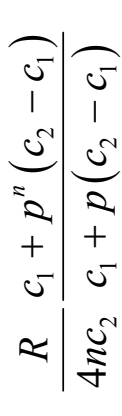 & 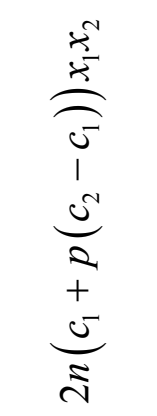 & 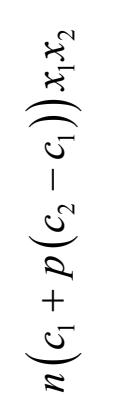 & 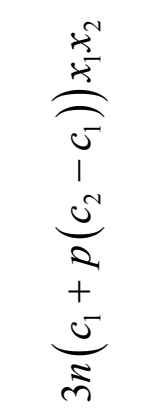 \\
\hline 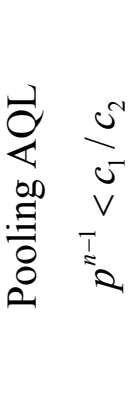 & $\approx \mid \underset{\Sigma}{\approx}$ & 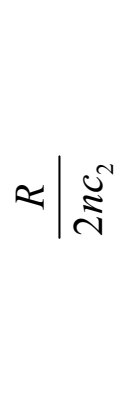 & 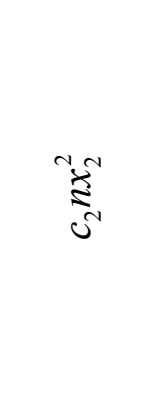 & $\begin{array}{l}\overbrace{\tilde{J}}^{N} \\
1 \\
\tilde{J}^{N} \\
\Xi\end{array}$ & $\begin{array}{l}\underset{\tilde{J}}{\tilde{J}} \\
1 \\
\mathfrak{J}^{N} \\
2 \\
+ \\
\underbrace{N} \\
\tilde{v}\end{array}$ \\
\hline 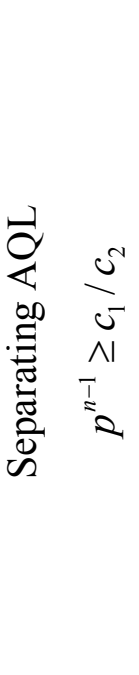 & 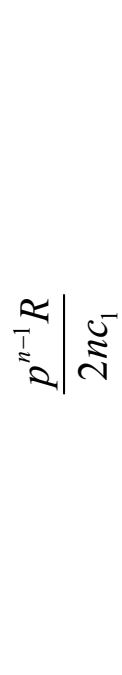 & 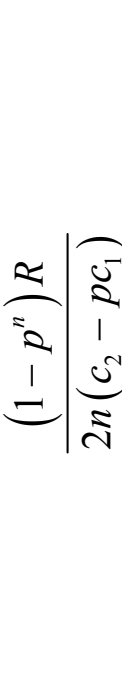 & 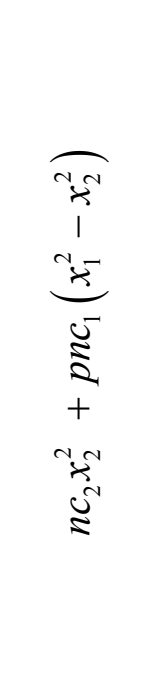 & 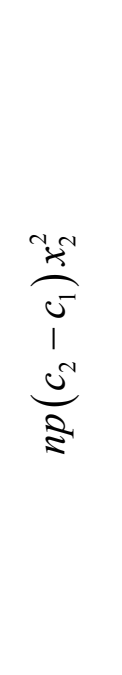 & 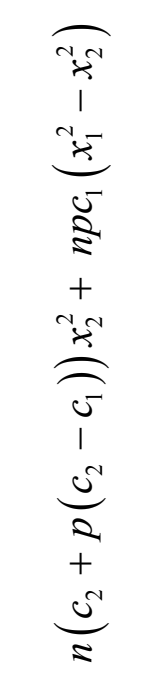 \\
\hline & $\begin{array}{l}\bar{x} \\
\stackrel{\vec{a}}{\bar{\Xi}} \\
\vec{\partial}\end{array}$ & 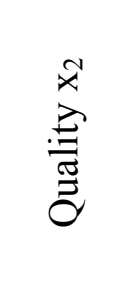 & 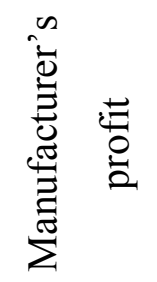 & 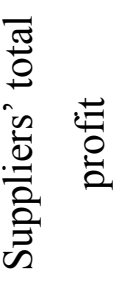 & 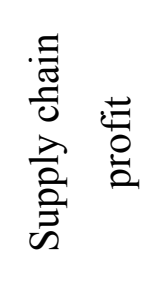 \\
\hline
\end{tabular}

